\title{
VITORIANO RUAS
}

\section{Méthodes d'éléments finis quasilinéaires en déplacement pour l'étude de milieux incompressibles}

RAIRO - Analyse numérique, tome 17, no 2 (1983), p. 161-194.

<http://www.numdam.org/item?id=M2AN_1983_17_2_161_0>

(C) AFCET, 1983, tous droits réservés.

L'accès aux archives de la revue «RAIRO - Analyse numérique » implique l'accord avec les conditions générales d'utilisation (http://www.numdam.org/ legal.php). Toute utilisation commerciale ou impression systématique est constitutive d'une infraction pénale. Toute copie ou impression de ce fichier doit contenir la présente mention de copyright.

\section{Numdam}




\title{
MÉTHODES D'ÉLÉMENTS FINIS QUASILINÉAIRES EN DÉPLACEMENT POUR LÉTUDE DE MILIEUX INCOMPRESSIBLES $(*)$
}

\author{
par Vitoriano RUAS
}

\author{
C.V.Communiqué par P. G. Ciarlet
}

\begin{abstract}
When incompressible fluid or elastic problems are approximated by finite element methods of displacement-pressure type, the question of obtaining well-posed discrete problems leading to convergent approximations is a delicate one. To achieve this aim, the space of approximate displacements is often excessively enlarged by comparison to the space of approximate pressures. In this paper, we propose the use of simplicial finite elements with a non-symmetric structure with respect to the barycenter of the simplex, which allow a substantial reduction of the displacement space with respect to the pressure space, yet which preserve optimal convergence properties as well as the uncoupling of the components of the displacements in the solution of the problem.
\end{abstract}

Résumé. - Lors de l'approximation par la méthode des éléments finis de type déplacementpression de problèmes de fluides ou d'élasticité incompressibles, la question d'obtenir des problèmes discrets bien posés, conduisant à une suite d'approximations convergentes est particulièrement délicate. C'est pour cela que cette exigence est assez souvent satisfaite en enrichissant excessivement l'espace de déplacements approchés, par rapport à l'espace de pressions choisi. Dans cet article on considère une méthode d'éléments finis simpliciaux, ayant une structure asymétrique par rapport au barycentre du simplexe, permettant de réduire autant que possible l'espace de déplacements par rapport à l'espace de pressions, de façon à ce que des propriétés de convergence optimales, ainsi que la possibilité de résoudre le problème par découplage des composantes du déplacement, soient préservées.

\section{Notation}

$\Omega$ étant un ouvert borné de $\mathbb{R}^{n}, \mathrm{n}=2$ ou 3 , de frontière $\Gamma$, pour tout ouvert $\mathrm{D}$ contenu dans $\Omega$, on dénotera par $\|\cdot\|_{\mathrm{m}, \mathrm{D}},|\cdot|_{\mathrm{m}, \mathrm{D}}$ et $(., .)_{\mathrm{m}, \mathrm{D}}$ respectivement, la norme, la semi-norme et le produit scalaire habituels [1] de l'espace de Sobolev $\mathrm{H}^{\mathrm{m}}(\mathrm{D})$, avec $\mathrm{H}^{\theta}(\mathrm{D})=\mathrm{L}^{2}(\mathrm{D})$, et les modifications évidentes, lorsqu'il s'agira de $\left[\mathrm{H}^{\mathrm{m}}(\mathrm{D})\right]^{1}, 1 \in \mathbb{N}, 1>0$. Lorsque $\mathrm{D}$ est le domaine $\Omega$ lui-même, on supprimera cet indice.

Pour tout espace de fonctions $\mathrm{V}$ définies sur $\mathrm{D}, \underset{\sim}{\mathrm{V} \text { désignera l'espace de champs }}$ à $\mathrm{n}$ fonctions, dont chaque composante appartient à $\mathrm{V}$.

On dénotera par $x . y$ le produit scalaire euclidien de $x, y \in \mathbb{R}^{1}$, où en général 1

(*) Reçu en février 1982.

( $\left.{ }^{1}\right)$ Departamento de Informática, Pontifícia Universidade Católica do Rio de Janeiro, 22453 Rio de Janeiro, Brésil.

R.A.I.R.O. Analyse numérique/Numerical Analysis, 0399-0516/1983/161/\$ 5.00

(C) AFCET-Bordas-Dunod 
sera égal à $\mathrm{n}$ dans le cas de vecteurs $\underset{\sim}{\mathrm{x}}$ et $\underset{\sim}{\mathrm{y}}$ de $\mathbb{R}^{\mathrm{n}}$ et à $\mathrm{n}^{2}$ dans le cas de tenseurs $\underset{\approx}{\mathrm{x}}$ et $\underset{\approx}{\mathrm{y}} \mathrm{de} \mathbb{R}^{\mathrm{n} \times \mathrm{n}}$. La norme associée à ce produit scalaire sera dénotée par $|\cdot|$.

$$
\mathrm{L}_{0}^{2}(\Omega) \text { est le sous-espace de } \mathrm{L}^{2}(\Omega) \text { des fonctions f telles que } \int_{\Omega} \mathrm{f} \underset{\sim}{\mathrm{x}}=0 .
$$

\section{INTRODUCTION}

Le but de cet article est d'étudier une classe d'éléments finis simpliciaux introduite dans [16], pour la simulation numérique du comportement de milieux incompressibles. La caractéristique fondamentale de ces éléments est leur structure asymétrique par rapport au barycentre du simplex. Ceci permet, entre autres, d'approcher la condition d'incompressibilité dans l'espace de déplacements choisi, de façon plus précise que pour les méthodes mixtes classiques, alors qu'on peut établir des résultats de convergence comparables. Notons que cette propriété d'approximation reste vraie, même lorsque la condition d'incompressibilité est non linéaire, comme dans le cas de matériaux incompressibles en régime de grandes déformations. Ce dernier cas, par ailleurs, a fait l'objet du travail [18] et maintenant nous nous intéressons en particulier à l'analyse de convergence pour certains de ces éléments asymétriques, dans le cadre de l'approximation des problèmes linéaires, qu'on peut poser de la manière suivante :

$\Omega$ étant le domaine de $\mathbb{R}^{\mathrm{n}}, \mathrm{n}=2,3$, de frontière $\Gamma$, occupé par le milieu à étudier, on veut trouver $\underset{\sim}{u}$ minimisant sur un ensemble $\underset{\sim}{\mathrm{W}}$, une certaine fonctionnelle d'énergie $\mathrm{E}(\mathrm{V})$ qui dépend d'un champ de vecteurs $\underset{\sim}{\mathrm{v}}$ défini sur $\Omega$, sous la contrainte $\mathrm{H}(\underset{\sim}{\mathrm{v}})=\tilde{0}$ p.p. dans $\Omega, \underset{\sim}{\forall} \in \underset{\sim}{\mathbf{W}}$.

Dans un grand nombre de cas intéressants, aussi bien en Mécanique des fluides que des solides, on a $\mathrm{H}(\underset{\sim}{\mathrm{v}})=$ div $\underset{\sim}{\mathrm{v}}$, et la contrainte en question est la condition d'incompressibilité linéaire qui s'écrit donc :

$$
\operatorname{div} \underset{\sim}{\mathrm{v}}=0 \quad \text { p.p. dans } \Omega \text {. }
$$

C'est cette contrainte qui interviendra dans les problèmes qu'on va traiter.

Remarque : En élasticité non linéaire, lorsqu'il s'agira d'un régime de grandes déformations on aura $\mathrm{H}(\underset{\sim}{v})=\mathbf{J}[\underset{\sim}{\mathbf{x}}+\underset{\sim}{\mathbf{v}}(\underset{\sim}{\mathbf{x}})]-1$, $\mathrm{J}(\underset{\sim}{\mathbf{w}})$ dénotant le Jacobien de w. Si les déformations sont petites, comme c'est le cas de l'élasticité linéaire, cette expression peut être simplifiée et devient (1.1).

Afin de mieux s'orienter vers les résultats essentiels qu'on peut obtenir avec les méthodes d'éléments finis que nous allons étudier, on considérera l'approximation de deux problèmes modèle où $\mathrm{E}$ est de la forme suivante :

$$
\mathrm{E}(\underset{\sim}{\mathrm{v}})=\frac{1}{2} \mathrm{a}(\underset{\sim}{\mathrm{v}} \underset{\sim}{\mathrm{v}})-\mathrm{L}(\underset{\sim}{\mathrm{v}})
$$


avec a $: \underset{\sim}{\mathrm{V}} \times \underset{\sim}{\mathrm{V}} \rightarrow \mathbb{R}$ et $\mathrm{L} \in \underset{\sim}{\mathrm{V}^{\prime}}, \underset{\sim}{\mathrm{V}}$ étant un espace de Hilbert de champs de vecteurs définis sur $\Omega$, tels que :

$$
\underset{\sim}{\mathrm{W}}=\{\underset{\sim}{\mathrm{V}} \underset{\sim}{\mathrm{v}} \in \underset{\sim}{\mathrm{V}}, \operatorname{div} \underset{\sim}{\mathrm{v}}=0 \text { p.p. dans } \Omega\} .
$$

Pour chacun des problèmes modèle, le choix de $\mathrm{a}, \mathrm{L}$ et $\mathrm{V}$ est respectivement :

1) Problème de Stokes stationnaire

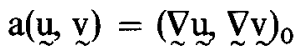

$$
\begin{aligned}
& \mathrm{L}(\underset{\sim}{\mathrm{v}})=(\underset{\sim}{\mathrm{f}}, \underset{\sim}{\mathrm{v}})_{0}, \quad \underset{\sim}{\mathrm{f}} \in{\underset{\sim}{\mathrm{L}}}^{2}(\Omega) \text { donné, et } \\
& \mathrm{V}=\mathrm{H}_{0}^{1}(\Omega) \text {. }
\end{aligned}
$$

2) Le problème de l'élasticité linéaire incompressible

$$
\mathrm{a}(\underset{\sim}{\mathrm{u}} \underset{\sim}{\mathrm{v}})=(\underset{\sim}{(\underset{\sim}{\mathrm{u}})}) \underset{\sim}{\varepsilon}(\underset{\sim}{\mathrm{v}}))_{0},
$$

où $\underset{\approx}{\varepsilon}$ est le tenseur de déformations défini par $\varepsilon_{\mathrm{ij}}(\mathrm{v})=\frac{1}{2}\left(\frac{\partial \mathrm{v}_{\mathrm{i}}}{\partial \mathrm{x}_{\mathrm{j}}}+\frac{\partial \mathrm{v}_{\mathrm{j}}}{\partial \mathrm{x}_{\mathrm{i}}}\right)$,

$$
\left.\mathrm{L}(\underset{\sim}{\mathrm{v}})=(\underset{\sim}{\mathrm{f}}, \underset{\sim}{\mathrm{v}})_{0}+\underset{\sim}{\mathrm{g}}, \underset{\sim}{\mathrm{v}}\right)_{0, \Gamma_{1}}, \underset{\sim}{\mathrm{f}} \in \underset{\sim}{\mathrm{L}^{2}}(\Omega) \text { donné },
$$

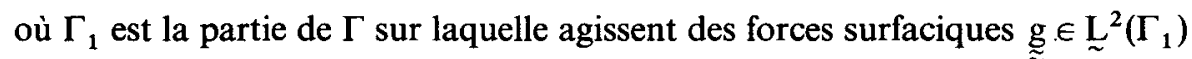
données, $\Gamma_{1} \neq \Gamma$, et

$$
\mathrm{V}=\left\{\mathrm{v} / \mathrm{v} \in \mathrm{H}^{1}(\Omega), \quad \mathrm{v}=0 \text { sur } \Gamma_{0}\right\},
$$

où $\Gamma_{0}$ est la partie fixée de $\Gamma$, avec $\Gamma_{0} \cap \Gamma_{1}=\emptyset$ et mes $\left(\Gamma_{0}\right) \neq 0$.

Remarque : Pour le problème de Stokes on peut considérer que $\Gamma=\Gamma_{0} \cup \Gamma_{1}$ $\operatorname{avec} \Gamma_{1}=\emptyset$.

Notons que pour ces deux problèmes, la solution $\underset{\sim}{u}$ satisfait respectivement aux équations suivantes (voir p. ex. [20] et [12]) :

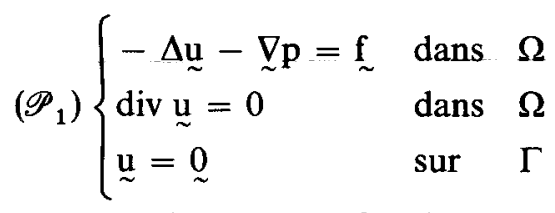

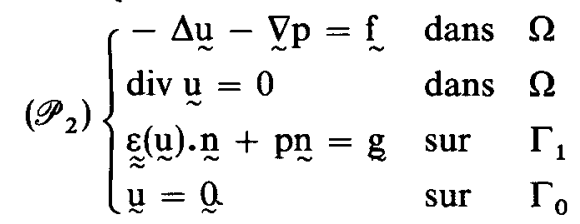

où $\mathbf{n}$ est le vecteur normal unitaire extérieur à $\Gamma_{1}$. 
Dans les deux cas p est un champ de pression à déterminer, associé à $u$, qui à son tour est un champ de vitesse dans le cas de $\left(\mathscr{P}_{1}\right)$ et un champ de déplacements dans le cas de $\left(\mathscr{P}_{2}\right)$. p est définie de manière unique pour $\left(\mathscr{P}_{2}\right)$ pourvu que mes $\left(\Gamma_{1}\right) \neq 0$. Lorsque mes $\left(\Gamma_{1}\right)=0$, p est définie à une constante additive près.

Nous allons considérer des méthodes d'approximation qui s'appliquent aux formulations mixtes des problèmes $\left(\mathscr{P}_{1}\right)$ et $\left(\mathscr{P}_{2}\right)$, dans lesquelles on s'affranchit en quelque sorte de la contrainte (1.1) par dualisation. Ceci est fait de la manière classique, à l'aide d'un multiplicateur de Lagrange appartenant à un espace $\mathrm{Q}$, qui n'est rien d'autre que la pression.

On sera ainsi amené à déterminer le point-selle $(\underset{\sim}{\mathrm{u}}, \mathrm{p}) \in \underset{\sim}{\mathrm{V}} \times \mathrm{Q}$ du Lagrangien $\mathscr{L}$ défini sur $\underset{\sim}{\mathrm{V}} \times \mathrm{Q}$, par

où

$$
\mathscr{L}(\underset{\sim}{\mathrm{q}} \mathrm{q})=\mathrm{E}(\underset{\sim}{\mathrm{v}})+\mathrm{b}(\underset{\sim}{\mathrm{v}} \mathrm{q})
$$

$$
\mathrm{b}(\mathrm{v}, \mathrm{q})=(\mathrm{q}, \mathrm{H}(\underset{\sim}{\mathrm{v}}))_{0}
$$

et

$\mathrm{Q}=\mathrm{L}_{0}^{2}(\Omega)$ si $\operatorname{mes}\left(\Gamma_{1}\right)=0$ et $\mathrm{Q}=\mathrm{L}^{2}(\Omega)$ si $\operatorname{mes}\left(\Gamma_{1}\right) \neq 0$.

Notons que pour la forme de $\mathrm{E}$ donnée par (1.2), (1.3) et (1.4), la détermination du point-selle de $\mathscr{L}$ équivaut à résoudre le problème suivant :

$$
\left(\mathscr{P}^{\prime}\right)\left\{\begin{array}{l}
\text { Trouver }(\underset{\sim}{\mathrm{u}}, \mathrm{p}) \in \underset{\sim}{\mathrm{V}} \times \mathrm{Q} \text { tel que } \\
\mathrm{a}(\underset{\sim}{\mathrm{u}}, \underset{\sim}{\mathrm{v}})+\underset{\sim}{\mathrm{b}}(\underset{\sim}{\mathrm{v}}, \mathrm{q})=\mathrm{L}(\underset{\sim}{\mathrm{v}}) \quad \underset{\sim}{\mathrm{v}} \in \underset{\sim}{\mathrm{V}} \\
\mathrm{b}(\underset{\sim}{\mathrm{u}}, \mathfrak{q})=0
\end{array}\right.
$$

Pour $\left(\mathscr{P}_{\mathrm{i}}\right), \mathrm{a}$, $\mathrm{L}$ et $\mathrm{V}$ sont donnés par $(1.3)_{\mathrm{i}},(1.4)_{\mathrm{i}}$ et $(1.5)_{\mathrm{i}} \mathrm{i}=1$, 2 , alors que $\mathrm{b}$ et $\mathrm{Q}$ sont définis par (1.6) et (1.7), respectivement.

La formulation $\left(\mathscr{P}^{\prime}\right)$ des problèmes $\left(\mathscr{P}_{1}\right)$ et $\left(\mathscr{P}_{2}\right)$ conduit tout naturellement à des méthodes d'éléments finis mixtes d'approximation. On va donc introduire deux espaces d'approximation ${\underset{\sim}{h}}_{h}$ et $Q_{h}$ de $\underset{\sim}{V}$ et $Q$, respectivement. Ceci étant, l'article se présente de la manière suivante :

Dans la Section 2 on définit de manière abstraite le problème approché et on donne des résultats de majoration de l'erreur d'approximation des pointsselle associés aux multiplicateurs de Lagrange, qui prennent en compte le cas où on utilise un espace non conforme pour la variable primale. Ceci parce que on considérera de tels espaces pour le cas tridimensionnel. Dans la Section 3, on introduit les méthodes asymétriques d'approximation, dont une étude de convergence est faite dans la Section 4. Enfin on conclut dans la Section 5 avec quelques remarques. 


\section{APPROXIMATION DES POINTS-SELLE NON CONFORME POUR LA VARIABLE PRIMALE}

On se donne deux espaces de Hilbert $V$ et $Q$ avec normes dénotées respectivement par $\|$. $\|$ et ]. . On représente par $\mathrm{L}(v)$ le produit de dualité de $\mathrm{L} \in V^{\prime}$ et $v \in V$, par $\mathrm{G}(q)$ celui de $\mathrm{G} \in Q^{\prime}$ et $q \in Q$ et on dénote par $(.,$.$) le produit$ scalaire dans $V$ auquel la norme $\|\cdot\|$ est associée.

Soit deux formes bilinéaires a et $\mathrm{b}$ avec $\mathrm{a}: V \times V \rightarrow \mathbb{R}$ et $\mathrm{b}: V \times Q \rightarrow \mathbb{R}$. On suppose qu'il existe des constantes strictement positives $A, \alpha, B$ et $\beta$ telles que :

$$
\begin{array}{rlrl}
\mathrm{a}(u, v) & \leqslant \mathrm{A}\|u\|\|v\| \quad \forall u, v \in V \\
\mathrm{a}(v, v) & \geqslant \alpha\|v\|^{2} & \forall v \in V \\
\mathrm{~b}(v, q) & \leqslant \mathrm{B}\|v\|\rfloor q \Gamma & \forall v \in V, \forall q \in Q \\
\sup _{v \in V} \frac{\mathrm{b}(v, q)}{\|v\|} \geqslant \beta q \Gamma & & \forall q \in Q
\end{array}
$$

cette dernière condition étant connue sous le nom de condition de Brezzi [3].

On sait [3] que sous ces hypothèses, pour $L \in V^{\prime}$ et $\mathrm{G} \in Q^{\prime}$ données, le problème de trouver $(u, p) \in V \times Q$ tel que :

$$
(\pi) \begin{cases}\mathrm{a}(u, v)+\mathrm{b}(v, p)=\mathrm{L}(v) & \forall v \in V \\ \mathrm{~b}(u, q)=\mathrm{G}(q) & \forall q \in Q\end{cases}
$$

admet une solution unique.

On va s'intéresser maintenant à l'approximation de $(\pi)$ moyennant l'introduction de deux familles d'espaces de dimension finie $\left(V_{\mathrm{h}}\right)_{\mathrm{h}}$ et $\left(Q_{\mathrm{h}}\right)_{\mathrm{h}}$, appelées à approcher $V$ et $Q$ avec $Q_{\mathrm{h}} \subset Q$ mais $V_{\mathrm{h}} \not \subset V \forall \mathrm{h}$. On suivra essentiellement la démarche de Thomas [21] pour le cas conforme, soit le cas où $V_{\mathrm{h}} \subset V \forall$ h.

Soit donc deux formes bilinéaires $\mathrm{a}_{\mathrm{h}}$ et $\mathrm{b}_{\mathrm{h}}$ avec $\mathrm{a}_{\mathrm{h}}: V_{\mathrm{h}} \times V_{\mathrm{h}} \rightarrow \mathbb{R}$ et $\mathrm{b}_{\mathrm{h}}$ : $V_{\mathrm{h}} \times Q_{\mathrm{h}} \rightarrow \mathbb{R}$, les deux formes étant définies aussi sur $V \times V$ et $V \times Q$ respectivement, de telle manière que :

$$
\begin{aligned}
& \mathrm{a}_{\mathrm{h}}(u, v)=\mathrm{a}(u, v) \quad \forall u, v \in V . \\
& \mathrm{b}_{\mathrm{h}}(v, q)=\mathrm{b}(v, q) \quad \forall v \in V \text { et } \forall q \in Q .
\end{aligned}
$$

On munit $V_{\mathrm{h}}$ d'un produit scalaire $(., .)_{\mathrm{h}}$ avec norme associée $\|\cdot\|_{\mathrm{h}}$, et on fait l'hypothèse suivante : 
$\exists A^{*}, \alpha^{*}, B^{*}$ et $\beta_{h}$, constantes strictement positives telles que :

$$
\begin{aligned}
\mathrm{a}_{\mathrm{h}}\left(u_{\mathrm{h}}, v_{\mathrm{h}}\right) & \leqslant \mathrm{A}^{*}\left\|u_{\mathrm{h}}\right\|_{\mathrm{h}}\left\|v_{\mathrm{h}}\right\|_{\mathrm{h}} & \forall u_{\mathrm{h}}, v_{\mathrm{h}} \in V_{\mathrm{h}}+V \\
\mathrm{a}_{\mathrm{h}}\left(v_{\mathrm{h}}, v_{\mathrm{h}}\right) \geqslant \alpha^{*}\left\|v_{\mathrm{h}}\right\|_{\mathrm{h}}^{2} & & \forall v_{\mathrm{h}} \in V_{\mathrm{h}} \\
\left.\mathrm{b}_{\mathrm{h}}\left(v_{\mathrm{h}}, q_{\mathrm{h}}\right) \leqslant \mathrm{B}^{*}\left\|v_{\mathrm{h}}\right\|_{\mathrm{h}}\right\rfloor q_{\mathrm{h}} \Gamma & & \forall v_{\mathrm{h}} \in V_{\mathrm{h}} \text { et } \forall q_{\mathrm{h}} \in Q \\
\left.\sup _{v_{\mathrm{h}} \in V_{\mathrm{h}}} \frac{\mathrm{b}_{\mathrm{h}}\left(v_{\mathrm{h}}, q_{\mathrm{h}}\right)}{\left\|v_{\mathrm{h}}\right\|_{\mathrm{h}}} \geqslant \beta_{\mathrm{h}}\right\rfloor q_{\mathrm{h}} \Gamma & & \forall q_{\mathrm{h}} \in Q_{\mathrm{h}}
\end{aligned}
$$

cette dernière condition étant la condition de Brezzi discrète.

Notons que, tout comme $\beta_{h}, A^{*}, B^{*}$ et $\alpha^{*}$ peuvent dépendre de $h$. Cependant, dans les applications qu'on va considérer, ces trois constantes seront indépendantes de $h$.

Sous ces hypothèses, si $\mathrm{L}_{\mathrm{h}}$ est une forme linéaire continue sur $V_{\mathrm{h}}$ qui approche $\mathrm{L}$, comme pour le cas de $(\pi)$, le problème approché de trouver $\left(u_{\mathrm{h}}, p_{\mathrm{h}}\right) \in V_{\mathrm{h}} \times Q_{\mathrm{h}}$ tel que :

$$
\left(\pi_{\mathrm{h}}\right) \begin{cases}\mathrm{a}_{\mathrm{h}}\left(u_{\mathrm{h}}, v_{\mathrm{h}}\right)+\mathrm{b}_{\mathrm{h}}\left(v_{\mathrm{h}}, p_{\mathrm{h}}\right)=\mathrm{L}_{\mathrm{h}}\left(v_{\mathrm{h}}\right) & \forall v_{\mathrm{h}} \in V_{\mathrm{h}} \\ \mathrm{b}_{\mathrm{h}}\left(u_{\mathrm{h}}, q_{\mathrm{h}}\right)=\mathrm{G}\left(q_{\mathrm{h}}\right) & \forall q_{\mathrm{h}} \in Q_{\mathrm{h}} .\end{cases}
$$

On supposera que la norme $\|\cdot\|_{\mathrm{h}}$ est définie sur $V$ aussi avec $\|v\|_{\mathrm{h}}=\|v\|$ $\forall v \in V$, et on s'appliquera à trouver des majorations de l'erreur $\left\|u-u_{\mathrm{h}}\right\|_{\mathrm{h}}$ et $\mathrm{J} p-p_{\mathrm{h}} \Gamma$. Pour ce faire, on introduit d'abord les variétés linéaires suivantes :

$$
\begin{aligned}
& U^{\mathrm{G}}=\{v \in V / \forall q \in Q, b(v, q)=\mathrm{G}(q)\} \\
& U_{\mathrm{h}}^{\mathrm{G}}=\left\{v_{\mathrm{h}} \in V_{\mathrm{h}} / \forall q_{\mathrm{h}} \in Q_{\mathrm{h}}, \mathrm{b}_{\mathrm{h}}\left(v_{\mathrm{h}}, q_{\mathrm{h}}\right)=\mathrm{G}\left(q_{\mathrm{h}}\right)\right\}
\end{aligned}
$$

et les espaces

$$
\begin{aligned}
U & =\{v \in V / \forall q \in Q, \mathrm{~b}(v, q)=0\} \\
U_{\mathrm{h}} & =\left\{v_{\mathrm{h}} \in V / \forall q_{\mathrm{h}} \in Q_{\mathrm{h}}, \mathrm{b}_{\mathrm{h}}\left(v_{\mathrm{h}}, q_{\mathrm{h}}\right)=0\right\} .
\end{aligned}
$$

Avec ces définitions, il est clair d'après la formulation de $(\pi)$ et de $\left(\pi_{\mathrm{h}}\right)$ que $u \in U, u_{\mathrm{h}} \in U_{\mathrm{h}}$ et que l'on a :

$$
\begin{aligned}
& \mathrm{a}(u, v)=\mathrm{L}(v) \quad \forall v \in U \\
& \mathrm{a}_{\mathrm{h}}\left(u_{\mathrm{h}}, v_{\mathrm{h}}\right)=\mathrm{L}_{\mathrm{h}}\left(v_{\mathrm{h}}\right) \quad \forall v_{\mathrm{h}} \in U_{\mathrm{h}} .
\end{aligned}
$$

On va d'abord chercher une majoration pour $\left\|u-u_{\mathrm{h}}\right\|_{\mathrm{h}}$. Soit donc 
$w_{\mathrm{h}}=u_{\mathrm{h}}-v_{\mathrm{h}}$, où $v_{\mathrm{h}}$ est un élément quelconque de $V_{\mathrm{h}}$. On a $w_{\mathrm{h}} \in U_{\mathrm{h}}$ et on peut écrire :

$$
\begin{aligned}
\mathrm{a}_{\mathrm{h}}\left(w_{\mathrm{h}}, w_{\mathrm{h}}\right) & =\mathrm{a}_{\mathrm{h}}\left(u_{\mathrm{h}}-v_{\mathrm{h}}+u-u, w_{\mathrm{h}}\right)= \\
& =\mathrm{a}_{\mathrm{h}}\left(u-v_{\mathrm{h}}, w_{\mathrm{h}}\right)+\mathrm{a}_{\mathrm{h}}\left(u_{\mathrm{h}}, w_{\mathrm{h}}\right)-\mathrm{a}_{\mathrm{h}}\left(u, w_{\mathrm{h}}\right) \\
& =\mathrm{a}_{\mathrm{h}}\left(u-v_{\mathrm{h}}, w_{\mathrm{h}}\right)+\mathrm{L}_{\mathrm{h}}\left(w_{\mathrm{h}}\right)-\mathrm{b}_{\mathrm{h}}\left(w_{\mathrm{h}}, p\right)-\mathrm{b}_{\mathrm{h}}\left(w_{\mathrm{h}}, p_{\mathrm{h}}\right) \\
& +\mathrm{b}_{\mathrm{h}}\left(w_{\mathrm{h}}, p\right)-\mathrm{a}_{\mathrm{h}}\left(u, w_{\mathrm{h}}\right)
\end{aligned}
$$

d'où, d'après $(2.1)_{\mathrm{h}}$ et $(2.2)_{\mathrm{h}}$ :

$$
\begin{aligned}
\alpha^{*}\left\|w_{\mathrm{h}}\right\|_{\mathrm{h}}^{2} \leqslant \mathrm{~A}^{*}\left\|u-w_{\mathrm{h}}\right\|_{\mathrm{h}}\left\|w_{\mathrm{h}}\right\|_{\mathrm{h}}+\mathrm{b}_{\mathrm{h}}\left(w_{\mathrm{h}}, p-p_{\mathrm{h}}\right)+ \\
+ \\
+\mathrm{L}_{\mathrm{h}}\left(w_{\mathrm{h}}\right)-\mathrm{a}_{\mathrm{h}}\left(u, w_{\mathrm{h}}\right)-\mathrm{b}_{\mathrm{h}}\left(w_{\mathrm{h}}, p\right) .
\end{aligned}
$$

Comme $\mathrm{b}_{\mathrm{h}}\left(w_{\mathrm{h}}, p_{\mathrm{h}}\right)=\mathrm{b}_{\mathrm{h}}\left(w_{\mathrm{h}}, q_{\mathrm{h}}\right)=0 \forall q_{\mathrm{h}} \in \mathcal{Q}_{\mathrm{h}}$ on $\mathrm{a}$ :

$$
\begin{aligned}
\left\|w_{\mathrm{h}}\right\|_{\mathrm{h}} \leqslant \frac{\mathrm{A}^{*}}{\alpha^{*}}\left\|u-v_{\mathrm{h}}\right\|_{\mathrm{h}}+\frac{\left|\mathrm{b}_{\mathrm{h}}\left(w_{\mathrm{h}}, p-q_{\mathrm{h}}\right)\right|}{\alpha^{*}\left\|w_{\mathrm{h}}\right\|_{\mathrm{h}}}+ \\
\quad+\frac{\left|\mathrm{L}_{\mathrm{h}}\left(w_{\mathrm{h}}\right)-\mathrm{a}_{\mathrm{h}}\left(u, w_{\mathrm{h}}\right)-\mathrm{b}_{\mathrm{h}}\left(w_{\mathrm{h}}, p\right)\right|}{\alpha^{*}\left\|w_{\mathrm{h}}\right\|_{\mathrm{h}}} .
\end{aligned}
$$

En posant :

$$
\mathrm{E}_{\mathrm{h}}\left(u, p, w_{\mathrm{h}}\right)=\mathrm{L}_{\mathrm{h}}\left(w_{\mathrm{h}}\right)-\mathrm{a}_{\mathrm{h}}\left(u, w_{\mathrm{h}}\right)-\mathrm{b}_{\mathrm{h}}\left(w_{\mathrm{h}}, p\right),
$$

en appliquant l'inégalité triangulaire et en majorant $\left|\mathrm{b}_{\mathrm{h}}\left(w_{\mathrm{h}}, p-q_{\mathrm{h}}\right)\right|$ par (2.3) , on obtient enfin :

$$
\begin{aligned}
\left\|u-u_{\mathrm{h}}\right\|_{\mathrm{h}} \leqslant\left(1+\frac{\mathrm{A}^{*}}{\alpha^{*}}\right) & \inf _{v_{h} \in U_{h}}\left\|u-v_{\mathrm{h}}\right\|_{\mathrm{h}}+ \\
& \left.+\frac{\mathrm{B}^{*}}{\alpha^{*}} \inf _{v_{h} \in U_{h}}\right\rfloor p-q_{\mathrm{h}}\left\lceil+\sup _{w_{h} \in V_{h}} \frac{\left|\mathrm{E}_{\mathrm{h}}\left(u, p, w_{\mathrm{h}}\right)\right|}{\alpha^{*}\left\|w_{\mathrm{h}}\right\|_{\mathrm{h}}} .\right.
\end{aligned}
$$

Cherchons maintenant une majoration semblable pour $\rfloor p-p_{\mathrm{h}} \Gamma$.

On a $\forall q_{\mathrm{h}} \in Q_{\mathrm{h}}$ et $\forall v_{\mathrm{h}} \in V_{\mathrm{h}}$ :

$$
\begin{aligned}
\mathrm{b}_{\mathrm{h}}\left(v_{\mathrm{h}}, p_{\mathrm{h}}-q_{\mathrm{h}}\right) & =\mathrm{L}_{\mathrm{h}}\left(v_{\mathrm{h}}\right)-\mathrm{a}_{\mathrm{h}}\left(u_{\mathrm{h}}, v_{\mathrm{h}}\right)-\mathrm{b}_{\mathrm{h}}\left(v_{\mathrm{h}}, q_{\mathrm{h}}\right) \\
& =\mathrm{L}_{\mathrm{h}}\left(v_{\mathrm{h}}\right)-\mathrm{a}_{\mathrm{h}}\left(u-u+u_{\mathrm{h}}, v_{\mathrm{h}}\right)-\mathrm{b}_{\mathrm{h}}\left(v_{\mathrm{h}}, q_{\mathrm{h}}-p+p\right) \\
& =\mathrm{L}_{\mathrm{h}}\left(v_{\mathrm{h}}\right)-\mathrm{a}_{\mathrm{h}}\left(u, v_{\mathrm{h}}\right)-\mathrm{b}_{\mathrm{h}}\left(v_{\mathrm{h}}, p\right)+\mathrm{a}_{\mathrm{h}}\left(u-u_{\mathrm{h}}, v_{\mathrm{h}}\right)+\mathrm{b}_{\mathrm{h}}\left(v_{\mathrm{h}}, p-q_{\mathrm{h}}\right)
\end{aligned}
$$

vol. $17, \mathrm{n}^{\circ} 2,1983$ 
d'où

$$
\frac{\mathrm{b}_{\mathrm{h}}\left(v_{\mathrm{h}}, p_{\mathrm{h}}-q_{\mathrm{h}}\right)}{\left\|v_{\mathrm{h}}\right\|_{\mathrm{h}}} \leqslant \frac{\mathrm{E}_{\mathrm{h}}\left(u, p, v_{\mathrm{h}}\right)}{\left\|v_{\mathrm{h}}\right\|_{\mathrm{h}}}+\frac{\mathrm{a}_{\mathrm{h}}\left(u-u_{\mathrm{h}}, v_{\mathrm{h}}\right)+\mathrm{b}_{\mathrm{h}}\left(v_{\mathrm{h}}, p-q_{\mathrm{h}}\right)}{\left\|v_{\mathrm{h}}\right\|_{\mathrm{h}}} \forall v_{\mathrm{h}} \in V_{\mathrm{h}} .
$$

Mais d'après $(2.4)_{\mathrm{h}},(2.1)_{\mathrm{h}}$ et $(2.3)_{\mathrm{h}}$ on a :

$$
\left.\beta_{\mathrm{h}}\right\rfloor p_{\mathrm{h}}-q_{\mathrm{h}}\left\lceil\leqslant \mathrm{A}^{*}\left\|u-u_{\mathrm{h}}\right\|_{\mathrm{h}}+\mathrm{B}^{*}\right\rfloor p-q_{\mathrm{h}} I+\sup _{w_{\mathrm{h}} \in V_{\mathrm{h}}} \frac{\left|\mathrm{E}_{\mathrm{h}}\left(u, p, w_{\mathrm{h}}\right)\right|}{\left\|w_{\mathrm{h}}\right\|_{\mathrm{h}}}
$$

d'où en déduit enfin :

$$
\begin{aligned}
& \rfloor p-p_{\mathrm{h}}\left\lceil\leqslant \frac{\mathrm{A}^{*}}{\beta_{\mathrm{h}}}\left\|u-u_{\mathrm{h}}\right\|_{\mathrm{h}}+\left(1+\frac{\mathrm{B}^{*}}{\beta_{\mathrm{h}}}\right) \inf _{q_{\mathrm{h}} \in Q_{\mathrm{h}}} \mid p-q_{\mathrm{h}}\lceil+\right. \\
& +\frac{1}{\beta_{\mathrm{h}}} \sup _{w_{\mathrm{h}} \in V_{\mathrm{h}}} \frac{\left|E_{\mathrm{h}}\left(u, p, w_{\mathrm{h}}\right)\right|}{\left\|w_{\mathrm{h}}\right\|_{\mathrm{h}}} .
\end{aligned}
$$

Maintenant en faisant des modifications évidentes dans un Lemme dû à Thomas [21], afin de tenir en compte l'indexation par $h$ des formes a et $b$ et de la norme $\|$.$\| , on peut s'affranchir de la restriction de l'appartenance de v_{\mathrm{h}}$ à $U_{\mathrm{h}}^{\mathrm{G}}$ dans la majoration (2.7), avec le résultat suivant :

LEMME $2.1: \mathrm{Si}(2.3)_{\mathrm{h}}$ et $(2.4)_{\mathrm{h}}$ sont vérifiées, on a la majoration :

$$
\inf _{v_{\mathrm{h}} \in U_{\mathrm{h}}}\left\|u-v_{\mathrm{h}}\right\|_{\mathrm{h}} \leqslant\left(1+\frac{\mathrm{B}^{*}}{\beta_{\mathrm{h}}}\right) \inf _{v_{\mathrm{h}} \in V_{\mathrm{h}}}\left\|u-v_{\mathrm{h}}\right\|_{\mathrm{h}} .
$$

Ce lemme et les majorations (2.7) et (2.8) nous permettent de déduire aisément le résultat suivant :

THÉORÈME 2.1 : Sous les hypothèses $(2.1)_{\mathrm{h}} \sim(2.4)_{\mathrm{h}}$, il existe des constantes $\mathrm{C}$ indépendantes de $\mathrm{h}\left({ }^{*}\right)$ telles que, si $\left(u_{\mathrm{h}}, p_{\mathrm{h}}\right)$ est la solution de $\left(\pi_{\mathrm{h}}\right)$ et $(u, p) \operatorname{de}(\pi)$, on $a$ :

$$
\begin{aligned}
\left\|u-u_{h}\right\|_{h} \leqslant \mathrm{C}\left[\left(1+\frac{1}{\beta_{h}}\right) \inf _{v h \in V_{h}}\left\|u-v_{h}\right\|_{h}+\right. \\
\left.\quad+\inf _{q_{h} \in Q_{h}}\right\rfloor-q_{h}\left\lceil+\sup _{w_{h} \in V_{h}} \frac{\left|E_{h}\left(u, p, w_{h}\right)\right|}{\left\|w_{h}\right\|_{h}}\right]
\end{aligned}
$$

(*) Ici comme partout ailleurs dans ce travail la lettre $C$ indexée ou non sera utilisée pour désigner les diverses constantes indépendantes du paramètre de discrétisation $h$. 


$$
\begin{aligned}
J p-q_{h}\left\lceil\leqslant \mathrm{C}\left[\frac{1}{\beta_{h}}\left\|u-u_{h}\right\|_{h}+\right.\right. \\
\left.\quad+\left(1+\frac{1}{\beta_{h}}\right) \inf _{q_{h} \in Q_{h}}\right\rfloor p-q_{h}\left\lceil+\frac{1}{\beta_{h}} \sup _{w_{h} \in V_{h}} \frac{\left|E_{h}\left(u, p, w_{h}\right)\right|}{\left\|w_{h}\right\|_{h}}\right]
\end{aligned}
$$

où $E_{h}\left(u, p, w_{h}\right)$ est donné par (2.6).

Remarquons que $\mathrm{E}_{\mathrm{h}}$ s'annule identiquement lorsque $V_{\mathrm{h}} \subset V$ et $\mathrm{L}=\mathrm{L}_{\mathrm{h}}$, et que dans ce cas (2.9) et (2.10) deviennent les majorations classiques pour les méthodes d'éléments finis mixtes conformes [21].

Maintenant on va appliquer ces résultats aux problèmes $\left(\mathscr{P}_{1}\right)$ et $\left(\mathscr{P}_{2}\right)$. Pour les deux cas la norme $\|\cdot\|$ devient $|\cdot|_{1}$ et $]$. I devient $|\cdot|_{0}$, alors que $\mathrm{G}=0$.

Soit $\mathcal{C}_{\mathrm{h}}$ une partition de $\Omega$ en éléments finis de diamètre maximal égal à $\mathrm{h}$ qui, outre les règles classiques d'assemblage, satisfait à certaines conditions spécifiques aux problèmes et méthodes qu'on considère dans ce travail, et qui seront précisées dans la Section 3.

Les espaces $V_{\mathrm{h}}$ et $Q_{\mathrm{h}}$ seront ainsi ${\underset{\sim}{\mathrm{h}}}_{\mathrm{h}}$ et $\mathrm{Q}_{\mathrm{h}}$, espaces de champs et de fonctions régulières sur chaque élément $K$ de $\mathfrak{C}_{\mathrm{h}}$, où $\mathrm{Q}_{\mathrm{h}} \subset \mathrm{Q}$. Maintenant, si ${\underset{\sim}{\mathrm{h} / \mathrm{K}}}_{\boldsymbol{\sim}} \in \mathrm{H}^{\mathbf{1}}(\mathrm{K})$ $\forall \mathrm{v}_{\mathrm{h}} \in \underline{\sim}_{\mathrm{h}}$ et $\forall \mathrm{K} \in \mathscr{C}_{\mathrm{h}}$, on pose pour $\left(\mathscr{P}_{1}\right)$ et $\left(\mathscr{P}_{2}\right)$ respectivement :

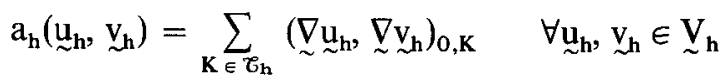

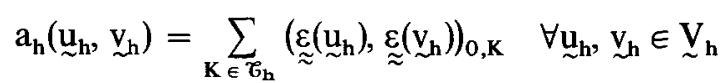

alors que pour les deux problèmes on aura :

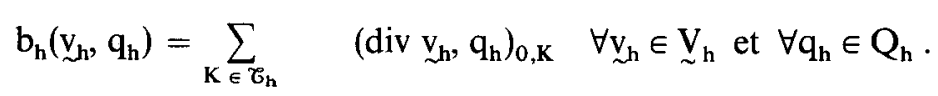

On supposera d'autre part que l'on définit bien un produit scalaire $(., .)_{\mathrm{h}}$ sur $\underset{\sim}{\mathrm{V}}$ aussi bien dans le cas de $\left(\mathscr{P}_{1}\right)$ que dans celui de $\left(\mathscr{P}_{2}\right)$, par :

$$
\left({\underset{\sim}{h}}_{h}, \underline{v}_{h}\right)_{h}=a_{h}\left(\underline{u}_{h}, \underline{\sim}_{h}\right)
$$

où $\mathrm{a}_{\mathrm{h}}$ est donné par $(2.11)_{1}$.

Dans ce cas, la norme $\|\cdot\|_{h}$ de ${\underset{\sim}{\mathrm{h}}}_{\mathrm{h}}$ sera donnée par l'expression suivante :

$$
\left\|v_{h}\right\|_{h}=\left[\sum_{K \in \mathcal{V}_{h}}\left|v_{h}\right|_{0, K}^{2}\right]^{1 / 2} \text {. }
$$


Notons maintenant que même si ${\underset{\sim}{\mathrm{h}}}_{\mathrm{h}} \not \subset \underset{\sim}{\mathrm{V}}$, L est définie sur ${\underset{\sim}{\mathrm{h}}}_{\mathrm{h}}$. On peut donc se passer de forme linéaire approchée $\mathrm{L}_{\mathrm{h}}$ et, en tenant compte des définitions $(2.11)_{\mathrm{i}},(2.12)$ et $(1.4)_{\mathrm{i}}$ de $\mathrm{a}, \mathrm{b}$ et $\mathrm{L}, \mathrm{i}=1,2$, le problème $\left(\pi_{\mathrm{h}}\right)$ est l'approximation $\left(\mathscr{P}_{\mathrm{h}}^{\prime}\right)$ de $\left(\mathscr{P}^{\prime}\right)$ (donc de $\left(\mathscr{P}_{1}\right)$ et $\left.\left(\mathscr{P}_{2}\right)\right)$.

$\left(\mathscr{P}_{\mathrm{h}}^{\prime}\right)$ consiste donc à trouver $\left({\underset{\sim}{\mathrm{h}}}_{\mathrm{h}}, \mathrm{p}_{\mathrm{h}}\right){\underset{\sim}{\mathrm{h}}}_{\mathrm{V}} \times \mathrm{Q}_{\mathrm{h}}$ tel que :

$$
\left(\mathscr{P}_{h}^{\prime}\right) \begin{cases}a_{h}\left(u_{h}, v_{h}\right)+b_{h}\left(v_{h}, p_{h}\right)=L\left(v_{h}\right) & \forall v_{h} \in{\underset{\sim}{V}}_{h} \\ b_{h}\left(u_{h}, q_{h}\right)=0 & \forall q_{h} \in Q_{h} .\end{cases}
$$

Notons tout de suite que, d'après les définitions $(2.11)_{i}$ et (2.12), et pourvu que $\|$. $\|_{h}$ donné par (2.14) soit effectivement une norme sur ${\underset{\sim}{h}}_{h},(2,1)_{h},(2.2)_{h}$ et $(2.3)_{\mathrm{h}}$ sont trivialement satisfaites. Il restera donc à vérifier $(2.4)_{\mathrm{h}}$ pour chaque ${\underset{\sim}{h}}_{h}$ et $\mathrm{Q}_{\mathrm{h}}$ choisis, afin de pouvoir appliquer les majorations du Théorème 2.1. Notons également que dans (2.9) et $(2.10)$ les deux premiers termes du second membre s'estiment de façon classique en théorie de l'approximation, alors que, s'il y a lieu, il restera à trouver des majorations convenables pour le dernier terme, dit de non-conformité, de façon à s'assurer de la convergence lorsque $\mathrm{h}$ tend vers zéro.

\section{LES ÉLEMENTS QUASI LINÉAIRES ASYMÉTRIQUES}

On supposera désormais que $\Omega$ est un domaine polyédrique et que si $\Gamma_{1} \neq 0$ pour $\mathrm{n}=3, \bar{\Gamma}_{1} \cap \bar{\Gamma}_{0}$ est un ensemble de lignes polygonales de $\mathbb{R}^{3}$. Nous supposons également que toute partition $\mathfrak{C}_{\mathrm{h}}$ est construite de telle manière que, aussi bien $\Gamma_{1}$ que $\Gamma_{0}$, peuvent être représentés en tant qu'union de faces d'éléments de $\mathfrak{C}_{\mathrm{h}}$.

Soit maintenant $h_{K}$ le diamètre de la sphère circonscrite et $\rho_{K}$ le diamètre de la sphère inscrite dans l'élément $K, K \in \mathcal{C}_{h}$. On pose $h=\max _{K \in \mathcal{C}_{h}} h_{K}$ et :

$$
c_{\mathbf{h}}=\min _{\mathbf{K} \in \mathcal{K}_{\mathbf{h}}} \frac{\rho_{\mathbf{K}}}{h_{\mathbf{K}}}
$$

et on suppose qu'il existe $c>0$ tel que $c_{h}>\forall h$, ce qui revient à dire que $\left\{\mathscr{C}_{\mathrm{h}}\right\}_{\mathrm{h}}$ est une famille de partitions régulières au sens classique.

Pour chaque $\mathscr{C}_{h}$ on définira $Q_{h}$ comme étant l'espace de fonctions $q_{h}$ qui sont constantes sur chaque élément de $\mathscr{C}_{\mathrm{h}}$ et qui de plus satisfont à la condition $\int_{\Omega} \mathrm{q}_{\mathrm{h}} \mathrm{dx}=0$, si mes $\left(\Gamma_{1}\right)=0$. On adoptera comme convention que les degrés de liberté de $\mathrm{Q}_{\mathrm{h}}$ sont les valeurs de la fonction au barycentre $\mathrm{G}$ des éléments de $\mathfrak{C}_{\mathrm{h}}$. 
Maintenant, on définit $V_{h}$ comme étant l'espace de fonctions dont la restriction sur chaque simplex $\mathrm{K} \in \mathcal{C}_{\mathrm{h}}$ appartient à un espace $\mathrm{P}_{a}$ défini comme suit :

Soient $S_{i}$ les sommets d'un simplex $K \in \mathscr{C}_{h}, i=1,2, \ldots, n+1$. Tout d'abord on attribue à $K$ une face privilégiée, à savoir la face opposée au sommet $S_{n+1}$. Cette face sera appelée la base $\mathbf{B}^{\mathrm{K}}$ de $\mathrm{K}$ et les faces $\mathrm{F}_{\mathrm{i}}^{K}$ opposées aux sommets $S_{i}$ pour $\mathrm{i}=1,2, \ldots, \mathbf{n}$ seront appelées les faces latérales de $\mathbf{K}$.

Soit maintenant $\lambda_{i}$ la coordonnée barycentrique de $K$ associée au sommet $\mathrm{S}_{\mathrm{i}}, \mathrm{i}=1,2, \ldots, \mathrm{n}+1$ et $\mathrm{S}_{\mathrm{n}+2}$ le barycentre de $\mathbf{B}^{\mathrm{K}}$. $\mathrm{P}_{a}$ est défini comme étant l'espace de dimension $n+2$ engendré par les $n+1 \lambda_{i}^{\prime} s$ et $\varphi$, où :

$$
\varphi=\sum_{\substack{j, \mathbf{k}=1 \\ \mathbf{j}<\mathbf{k}}} \lambda_{\mathbf{j}} \lambda_{\mathbf{k}} .
$$

On peut vérifier que l'ensemble de degrés de liberté $\left\{a_{i}\right\}_{i=1}^{n+2}$, où $a_{i}$ est la valeur de la fonction au point $S_{\mathrm{i}}$, est $\mathrm{P}_{a}$-unisolvent et que les fonctions de base associées sont données par :

$$
\left.\begin{array}{l}
\mathrm{p}_{\mathrm{i}}=\lambda_{\mathrm{i}}-\frac{2}{\mathrm{n}-1} \varphi \\
\mathrm{p}_{\mathrm{n}+1}=\lambda_{\mathrm{n}+1} \\
\mathrm{p}_{\mathrm{n}+2}=3 \varphi .
\end{array}\right\}
$$

Sur la figure 3.1, on donne une illustration des éléments asymétriques ainsi définis, où $O$ représente les degrés de liberté de $\bigvee_{h}$ et $\times$ ceux de $Q_{h}$.

On remarquera que les inclusions $\mathrm{P}_{1} \subset \mathrm{P}_{a} \subset \mathrm{P}_{2}$ sont satisfaites, $\mathrm{P}_{\mathrm{k}}$ étant l'espace de polynômes de degré inférieur ou égal à $\mathrm{k}$, définis sur $\mathrm{K}\left({ }^{*}\right)$.
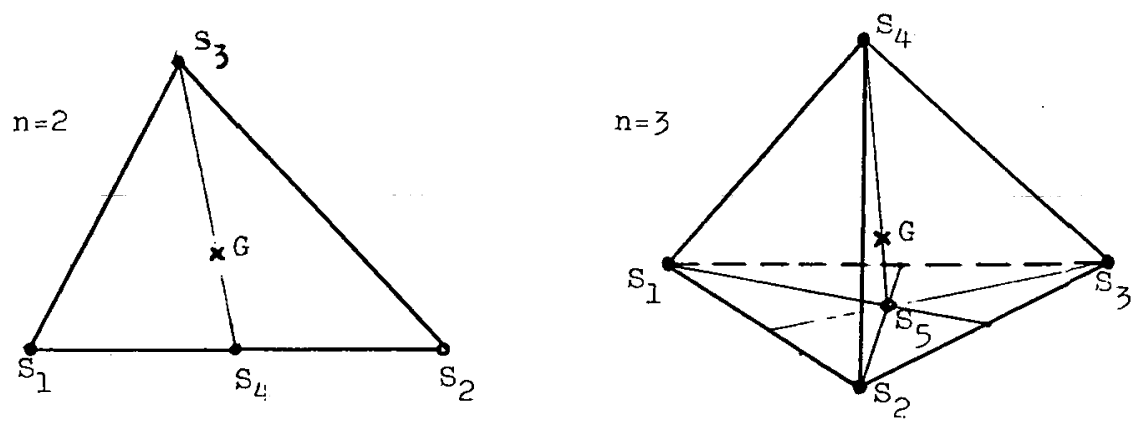

Figure 3.1. - Les éléments quasi linéaires asymétriques.

$\left(^{*}\right)$ Comme dans [16], a peut être interprété comme un indice rationnel. Dans le cas présent, on aurait $a=\left(C_{n+1}^{2}+1\right) / C_{n+1}^{2}$.

vol. $17, \mathrm{n}^{\circ} 2,1983$ 
Il doit être clair qu'en raison de la structure asymétrique de $\mathrm{P}_{a}$, on ne peut pas espérer associer ces éléments asymétriques à des partitions arbitraires de $\Omega$. En effet $\mathcal{C}_{\mathrm{h}}$ doit respecter la condition de compatibilité suivante :

Toute face commune à deux simplexes de $\mathfrak{C}_{\mathrm{h}}$ doit être pour les deux simplexes, soit une base, soit une face latérale.

Maintenant, pour construire des $\mathfrak{C}_{\mathrm{h}}$ qui satisfont à la condition ci-dessus, ce qu'on appellera une partition compatible, il suffit de pouvoir décider sans équivoque des faces qui seront les bases, de façon à ce que chaque élément n'ait qu'une base. Or, en pratique ceci n'est nullement difficile à réaliser, et ne présente aucune contrainte pour le calcul avec ces éléments. A titre d'exemple nous proposons ci-dessus deux constructions simples de partitions compatibles de domaines quelconques, avec lesquelles on va travailler par la suite, parmi de nombreuses autres possibilités :

Partition $\mathfrak{C}_{\mathrm{h}}^{1}$ : Dans le cas bidimensionnel on construit d'abord $\mathfrak{C}_{\mathrm{h}}$, une partition de $\Omega$ en quadrilatères convexes (comme pour l'élément bilinéaire isoparamétrique $Q_{1}$ ). Ensuite on subdivise chaque quadrilatère en deux triangles par une diagonale $\mathrm{AB}$ quelconque. Ces diagonales seront les seules bases des éléments de la triangulation $\mathcal{C}_{\mathrm{h}}^{1}$ ainsi générée (voir fig. 3.2a).

Dans le cas tridimensionnel on construit d'abord $C_{\mathrm{h}}$, partition arbitraire de $\Omega$ en hexaèdres convexes ayant des faces quadrilatérales (ici aussi, comme pour l'élément trilinéaire isoparamétrique $Q_{1}$ ). Ensuite, chaque hexaèdre est subdivisé en cinq tétraèdres de la manière classique indiquée sur la figure $3.2 b$. Enfin le tétraèdre $A B C D$ situé au milieu de l'hexaèdre est coupé en quatre tétraèdres, en joignant son barycentre $\mathrm{E}$ à $\mathrm{A}, \mathrm{B}, \mathrm{C}$ et $\mathrm{D}$. Les seules bases des éléments de la partition en tétraèdres ainsi construite, sont les faces des tétraèdres $\mathrm{ABCD}$ (en fait dans chaque hexaèdre de $\mathcal{C}_{\mathrm{h}}$ on a quatre paires de tétraèdres de $\mathcal{C}_{\mathrm{h}}^{1}$ ayant une base commune).

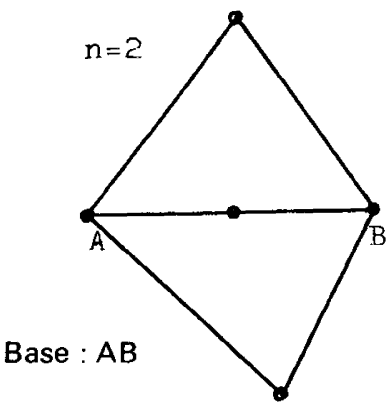

$3.2 \mathrm{a}$

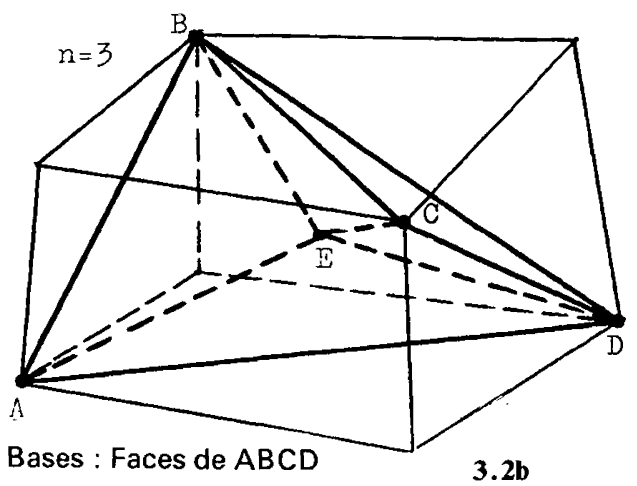

3.2b

Figure 3.2. - Construction des partitions compatibles $\mathcal{C}_{\mathrm{h}}^{1}$. 
Partition $\mathscr{C}_{\mathrm{h}}^{2}$ : On construit d'abord $\mathscr{C}_{\mathrm{h}}$, partition arbitraire de $\Omega$ en n-simplexes $K$. Ensuite chaque $K \in \mathscr{C}_{h}$ est subdivisé en $n+1$ simplexes en joignant son barycentre $\mathrm{G}$ à ses $\mathrm{n}+1$ sommets (voir fig. 3.3). Cette sous-partition devient la partition compatible $\mathcal{G}_{\mathrm{h}}^{2}$ si les bases de celle-ci sont définies comme étant les faces des simplexes de $\mathfrak{G}_{\mathrm{h}}$.

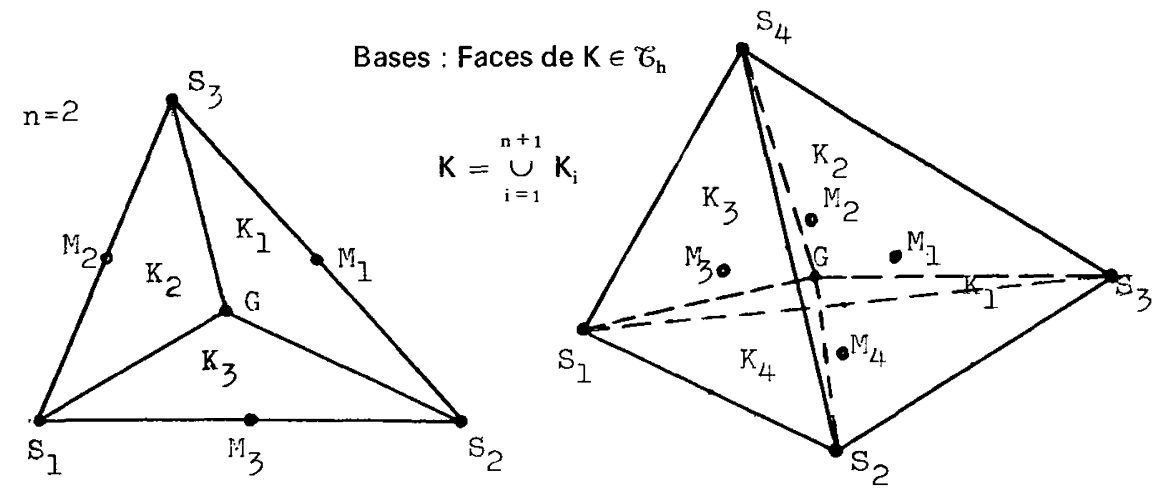

Figure 3.3. - Construction des partitions compatibles $\mathcal{C}_{\mathrm{h}}^{2}$.

D'une façon générale, une fois construite une partition compatible, on définit les degrés de liberté de $\mathrm{V}_{\mathrm{h}}$ comme étant les valeurs de la fonction aux sommets et aux barycentres des bases des éléments de cette partition, exceptées les valeurs aux nœuds situés sur $\Gamma_{0}$, où toute fonction de $V_{h}$ s'annule nécessairement.

Ceci étant, compte tenu de la continuité d'une fonction de $\mathrm{V}_{\mathrm{h}}$ aux nœuds associés aux degrés de liberté, on peut dire que ${\underset{\sim}{h}}_{h} \subset \underset{\sim}{V}$ pour $n=2$. Cependant si $\mathrm{n}=3$ cette inclusion n'est plus vérifiée et l'élément est donc non conforme. En effet, le simple examen de (3.1) et (3.2) indique qu'une fonction de $V_{h}$ n'est nécessairement continue que sur les bases de la partition. Néanmoins, on va prouver que la partie du flux associé à $\varphi$ (la composante non conforme de $\mathrm{P}_{a}$ ) qui porte sur les faces latérales de $\mathrm{K}$, correspond à une fraction fixe du flux de cette même composante à travers la base. Or, justement sur la base on a continuité, ce qui permettra de démontrer des résultats de convergence dans la section suivante.

LEMME 3.1 : Soit $\mathrm{K}$ un tétraèdre et $\mathrm{n}_{\mathrm{K}}$ le vecteur unitaire normal extérieur $\grave{a} \partial \mathrm{K}$, la frontière de $\mathrm{K}$. Soit $\underset{\sim}{\Psi}$ le champ de vecteurs défini sur $\mathrm{K}$, tel que $\underset{\sim}{\Psi}=\underset{\sim}{\beta} \varphi$, $\underset{\sim}{\beta} \in \mathbb{R}^{3}, \varphi$ étant donné par (3.1). Alors on $a:$

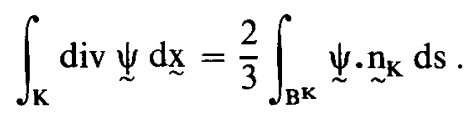

vol. $17, \mathrm{n}^{\circ} 2,1983$ 
Démonstration : D'après la formule de Stokes on a :

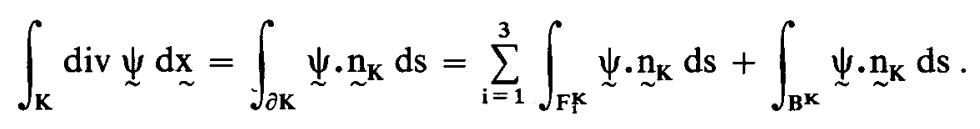

Comme $\varphi / F_{i}^{K}=\lambda_{j} \lambda_{k}$, avec $i, j$, et k distincts, on a :

$$
\sum_{\mathrm{i}=1}^{3} \int_{\mathrm{F}_{\mathrm{i}}^{\mathrm{K}}} \Psi \cdot \sim_{\sim}^{\mathrm{K}} \mathrm{ds}=\frac{1}{12} \sum_{\mathrm{i}=1}^{3} \underset{\sim}{\beta} \cdot \sim_{\mathrm{N}_{\mathrm{K} / \mathrm{F}_{\mathrm{i}}}} \text { aire }\left(\mathrm{F}_{\mathrm{i}}^{\mathrm{K}}\right)
$$

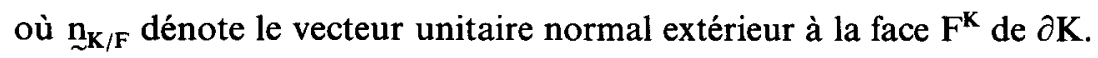

D'autre part, pour un vecteur constant $\underset{\sim}{\beta}$ on a :

$$
0=\int_{\mathbf{K}} \operatorname{div} \underset{\sim}{\beta} \underset{\sim}{\mathrm{dx}}=\sum_{\mathrm{i}=1}^{3} \underset{\sim}{\beta} \cdot{\underset{\sim}{\mathrm{K} / \mathrm{F}_{\mathrm{i}}}} \operatorname{aire}\left(\mathrm{F}_{\mathbf{i}}^{\mathbf{K}}\right)+\underset{\sim}{\beta} \cdot{\underset{\sim}{\mathrm{K} / \mathbf{B}}} \operatorname{aire}\left(\mathrm{B}^{\mathrm{K}}\right) .
$$

d'on on déduit que

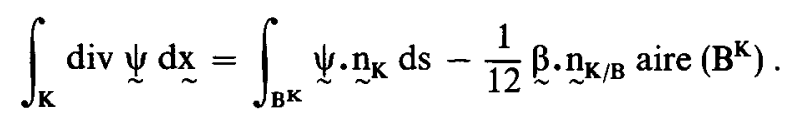

Enfin, (3.3) est la conséquence du fait que

$$
\int_{\mathrm{B}^{\mathrm{K}}} \underset{\sim}{\Psi} \underset{\sim}{\underset{\sim}{\mathrm{n}_{\mathrm{K}}}} \mathrm{ds}=\frac{1}{4} \underset{\sim}{\beta} \cdot{\underset{\sim}{\mathrm{K} / \mathrm{B}}}_{\mathrm{B}} \text { aire }\left(\mathrm{B}^{\mathrm{K}}\right) . \quad \text { c.q.f.d. }
$$

\section{RÉSUltats DE CONVERgENCE}

Dans ce chapitre on va donner une analyse de convergence pour les problèmes $\left(\mathscr{P}_{1}\right)$ et $\left(\mathscr{P}_{2}\right)$, relative à l'approximation par la méthode d'éléments finis décrite dans la section précédente.

On rappelle que la difficulté essentielle pour établir l'existence et l'unicité de la solution de $\left(\mathscr{P}_{h}^{\prime}\right)$, ainsi que la convergence de $\left({\underset{\sim}{h}}_{h}, p_{h}\right)$ vers $(\underset{\sim}{u}, p)$ consiste à démontrer l'inégalité $(2.4)_{h}$, avec une estimation de $\beta_{h}$ en fonction de $h$ aussi fine que possible.

Dans le cas des partitions de type $\mathcal{C}_{\mathrm{h}}^{1}$, on peut démontrer que la constante $\beta_{\mathrm{h}}$ dépend de $\mathrm{h}$ en général et que cette dépendance s'exprime exactement de la même manière que celle de la constante $\beta_{\mathrm{h}}$ relative à la méthode d'éléments finis étudiée par Pitkäranta [10] et [14] pour le problème de Stokes. Celle-ci est définie de la manière suivante : 
- $V_{h}$ est l'espace de fonctions continues, dont la restriction à chaque élément de la partition $\mathcal{C}$ (un quadrilatère ou un hexahèdre) à partir de laquelle $\mathscr{C}_{\mathbf{h}}^{1}$ est construite, appartient à l'espace $Q_{1}$ isoparamétrique (cf. [4]).

L'étude détaillée de cette équivalence fera l'objet d'un article de l'auteur en cours de préparation. Néanmoins, puisqu'on peut ramener l'analyse de convergence à celle de l'élément $\mathrm{Q}_{1} \times \mathrm{P}_{0}$ décrit ci-dessus, à titre d'illustration on donne un résultat, extrait de [10], qui s'applique au cas de notre élément $\mathrm{P}_{a} \times \mathrm{P}_{0}$ :

THÉORÈME 4.1 : Soit $\Omega$ un rectangle et $\Gamma_{1}=\emptyset$. Si on utilise une triangulation de type $\mathcal{C}_{\mathrm{h}}^{1}$ construite à partir d'un maillage uniforme de $\Omega$ en $\mathrm{M} \times \mathrm{N}$ rectangles, avec $\mathbf{M}$ et $\mathbf{N}$ pairs, alors on a :

$$
\left|\underset{\sim}{\mathbf{u}}-{\underset{\sim}{\mathrm{u}}}_{\mathrm{h}}\right|_{1} \leqslant \mathrm{Ch}\left[|\underset{\sim}{\mathbf{u}}|_{2}+|\mathrm{p}|_{1}+|\underset{\sim}{\mathbf{u}}|_{3, \mathrm{r}, \Omega}\right]
$$

pour tout réel $\mathrm{r}, \mathrm{r}>1$, où $|\cdot|_{3, \mathrm{r}, \Omega}$ dénote la semi-norme habituelle de l'espace de Sobolev $\mathrm{W}^{3, \mathrm{r}}(\Omega)(c f .[1])$.

Remarque : Pour ce qui est de la convergence de $\mathrm{p}_{\mathrm{h}}$ vers $\mathrm{p}$, on se réfère à l'article [10]. Des résultats analogues en dimension trois peuvent être trouvés dans [14].

Dans cet article, on considérera en détail le cas de la partition $\mathcal{C}_{\mathrm{h}}^{2}$, pour lequel on vérifie que $(2.4)_{h}$ est satisfaite avec $\beta_{h}=0\left(h^{2-s}\right)$, où $s$ est un réel appartenant à l'intervalle $[3 / 2-\varepsilon, 2]$, qui correspond à la régularité $H^{\mathrm{s}}(\Omega)$ de la solution du problème mêlé suivant :

$$
\left.\begin{array}{l}
-\Delta \mathrm{z}=\mathrm{f} \text { dans } \Omega \text { pour } \mathrm{f} \in \mathrm{L}^{2}(\Omega) \\
\frac{\partial \mathrm{z}}{\partial \mathrm{n}}=0 \operatorname{sur} \Gamma_{1} \\
\mathrm{z}=0 \text { sur } \Gamma_{0} .
\end{array}\right\}
$$

D'après les résultats de Grisvard [9] et Raugel [15], dans le cas bidimensionnel on a le résultat suivant :

Soit $\Omega$ un polygone convexe et $\left\{A_{i}\right\}_{i=1}^{1}$ l'ensemble de points où $\bar{\Gamma}_{1}$ intersecte $\bar{\Gamma}_{0}$. Si $\Gamma_{1}$ est vide, on prend $I=0$. On associe à $A_{i}$ un nombre $\alpha_{i}$, tel que si $A_{i}$ est un sommet de $\Gamma, \pi / \alpha_{i}$ est l'angle du coin correspondant, et si $A_{i}$ n'est pas un sommet de $\Gamma, \alpha_{i}=1$.

Alors on a $\mathbf{z} \in \mathbf{H}^{\mathrm{s}}(\Omega)$ avec $\mathrm{s}=1+\alpha / 2-\delta$, où :

$$
\begin{aligned}
& \alpha=2 \text { et } \delta=0 \text { si } I=0 \\
& \alpha=\min \left[2, \max \left(1, \min _{1 \leqslant i \leqslant I} \alpha_{i}\right)\right] \text { et } \delta=\varepsilon \text { si } I>0 .
\end{aligned}
$$


En outre, il existe une constante $\mathrm{C}$ indépendante de $\mathrm{f}$ telle que :

$$
\|\mathrm{z}\|_{\mathrm{s}} \leqslant \mathrm{C}\|\mathrm{f}\|_{0} \text {. }
$$

Dans le cas tridimensionnel des résultats semblables sont donnés dans [9]. Cependant nous allons nous contenter de limiter.notre étude au cas où on peut affirmer que $\mathrm{z} \in \mathrm{H}^{2}(\Omega)$. La raison en est le fait que la non-conformité de l'élément tridimensionnel complique l'analyse considérablement dans le cas général, mais si $\mathrm{z} \in \mathrm{H}^{2}(\Omega)$ pour tout $\mathrm{f} \in \mathrm{L}^{2}(\Omega)$, alors le problème peut être simplifié. Notons, par ailleurs, que la régularité $\mathrm{H}^{2}(\Omega)$ peut être effectivement atteinte dans beaucoup de cas intéressants en pratique, et notamment pour le problème $\left(\mathscr{P}_{1}\right)$, lorsque $\Omega$ est convexe [11].

Une remarque qui s'impose à ce stade, est le fait qu'on peut prouver l'existence et l'unicité de la solution du problème $\left(\mathscr{P}_{\mathrm{h}}^{\prime}\right)$ indépendamment de la régularité de z (voir [18]).

En résumé, on supposera désormais tout simplement que $\Omega$ a une frontière polyédrique qui consiste en deux parties disjointes $\Gamma_{0}$ et $\Gamma_{1}$, avec mes $\left(\Gamma_{0}\right)$ non nulle, de telle sorte que la solution $\mathrm{z}$ de (4.1) appartient à $\mathrm{H}^{\mathrm{s}}(\Omega)$ pour quelque $\mathrm{s}, 3 / 2-\varepsilon \leqslant s \leqslant 2$, ainsi que la validité de (4.2).

En guise de préparation on donne les lemmes suivants :

Tout d'abord on rappelle un Lemme dû à Ciarlet [4] pour les formes bilinéaires :

LEMME 4.1 [4] : Soit $\sigma: \mathrm{H}^{\mathrm{k}+1}(\mathrm{D}) \times \mathrm{P} \rightarrow \mathbb{R}$ une forme bilinéaire, $\mathrm{k} \in \mathbb{N}$, avec $\mathrm{P}_{1} \subset \mathrm{P} \subset \mathrm{H}^{1+1}(\mathrm{D}), 1 \in \mathbb{N}$, $\mathrm{P}$ étant normé par $\|\cdot\|_{1+1, \mathrm{D}}$. Alors si $\sigma$ satisfait

$$
\begin{aligned}
& \|\sigma\|=\sup _{(\mathbf{y}, \mathbf{w}) \in \mathbf{H}^{\mathrm{k}} \mathbf{1}_{1}(\mathbf{D}) \times \mathbf{P}} \frac{\sigma(\mathrm{y}, \mathrm{w})}{\|\mathrm{y}\|_{\mathrm{k}+1, \mathbf{D}}\|\mathbf{w}\|_{1+1, \mathrm{D}}}<\infty \\
& \sigma(\mathrm{y}, \mathrm{w})=0 \quad \forall \mathrm{y} \in \mathrm{H}^{\mathrm{k}+1}(\mathrm{D}) \quad \forall \mathrm{w} \in \mathrm{P}_{1} \\
& \sigma(\mathrm{y}, \mathrm{w})=0 \quad \forall \mathrm{y} \in \mathbf{P}_{\mathrm{k}} \quad \forall \mathrm{w} \in \mathbf{P}
\end{aligned}
$$

il existe une constante $C$ qui ne dépend que de $D$, telle que :

$|\sigma(\mathrm{y}, \mathrm{w})| \leqslant \mathrm{C}\|\sigma\||\mathrm{y}|_{\mathrm{k}+1, \mathrm{D}}|\mathrm{w}|_{1+1, \mathrm{D}} \quad \forall(\mathrm{y}, \mathrm{w}) \in \mathrm{H}^{\mathrm{k}+1}(\mathrm{D}) \times \mathbf{P}$.

On se bornera momentanément au cas tridimensionnel.

LEMME 4.2 : Soit $\mathrm{n}=3$ et $\left\{\mathcal{G}_{\mathrm{h}}\right\}_{\mathrm{h}}$ une famille régulière de partitions en tétraèdres de $\Omega$. Soit $\underset{\sim}{\mathrm{z}}$ un champ de vecteurs tels que $\mathrm{z}_{\mathrm{i}}$ est la solution de (4.1) pour $\mathrm{f}=\mathrm{f}_{\mathrm{i}}, \underset{\sim}{\mathrm{f}}=\left(\mathrm{f}_{1}, \mathrm{f}_{2}, \ldots, \mathrm{f}_{n}\right) \in \underset{\sim}{\mathrm{L}^{2}}(\Omega)$. Alors si on peut affirmer que $\underset{\sim}{z} \underset{\sim}{H^{2}}(\Omega)$, on $a$ :

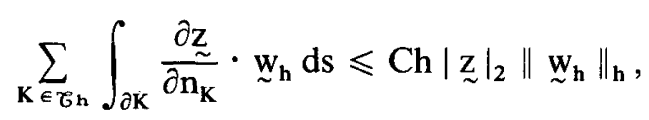


où $\partial \mathrm{g} / \partial \mathrm{n}_{\mathrm{K}}$ désigne la dérivée première normale extérieure d'une fonction $\mathrm{g}$, par rapport à la frontière $\partial \mathrm{K}$ de $\mathrm{K}$.

Démonstration : Comme $\underset{\sim}{\mathbf{z}} \in \underset{\sim}{\mathrm{H}^{2}}(\mathrm{~K}), \underset{\underset{\sim}{\mathrm{Z}}}{\partial \mathbf{n}_{\mathbf{K}}} \in \underset{\sim}{\mathrm{\sim}^{1 / 2}}(\mathrm{~F})$ pour toute face $\mathrm{F}$ de $\partial \mathrm{K}$, ce qui signifie que (4.3) a un sens.

On introduit maintenant l'opérateur $\Pi:{\underset{\sim}{h}}_{h} \rightarrow \underset{\sim}{C}(\bar{\Omega})$, tel que $\prod_{\sim} \underset{h / K}{ }$ est le $P_{1}$-interpolé de ${\underset{\sim}{\mathrm{h}}}_{\mathrm{h}}$ aux sommets de $\mathrm{K}, \forall \mathrm{K} \in \mathscr{C}_{\mathrm{h}}$. Compte tenu de la continuité de $\prod_{\sim} w_{h}$ sur toute face de $\mathscr{C}_{\mathrm{h}}$ et de sa nullité sur $\Gamma_{0}$ on a :

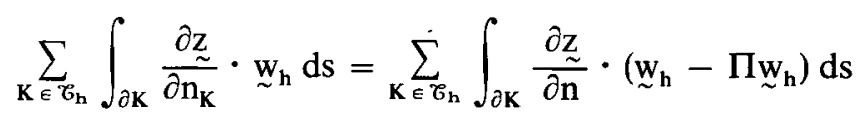

puisque $\partial \mathrm{z} / \partial \mathrm{n}=0$ sur $\Gamma_{1}$. De plus, on $\mathbf{a}$ :

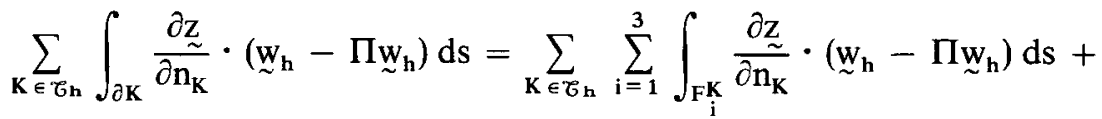

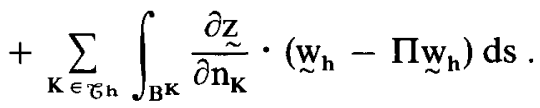

Comme $\underset{\sim}{\mathrm{h}}$ est continu sur les bases $B^{K}$ de $\mathcal{C}_{\mathrm{h}}$, le deuxième terme du second membre ci-dessus s'annule, et on peut réécrire :

$$
\begin{aligned}
& \sum_{\mathbf{K} \in \mathfrak{G}_{\mathbf{h}}} \int_{\partial \mathbf{K}} \frac{\partial \underset{\sim}{\mathbf{z}}}{\partial \mathrm{n}_{\mathbf{K}}} \cdot \underset{\sim}{w_{\mathbf{h}}} \mathrm{ds}=
\end{aligned}
$$

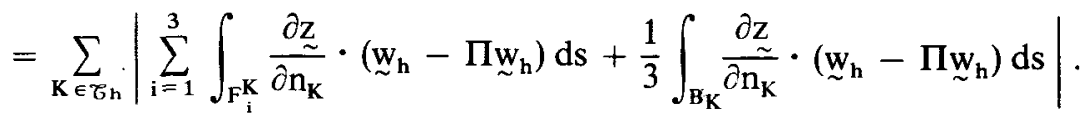

On considère maintenant la forme bilinéaire $\sigma_{K}: \mathrm{H}^{2}(\mathrm{~K}) \times \mathrm{P}_{a} \rightarrow \mathbb{R}$

$$
\sigma_{\mathrm{K}}(\mathrm{u}, \mathrm{w})=\frac{1}{3} \int_{\mathrm{B}^{\mathrm{K}}} \underset{\sim}{\nabla \mathrm{u}} \cdot \sim_{\sim}^{n_{K}}(\mathrm{w}-\Pi \mathrm{w}) \mathrm{ds}+\sum_{\mathrm{i}=1}^{3} \int_{\mathrm{F}_{\mathrm{i}}} \underset{\sim}{\nabla \mathrm{u}} \cdot{\underset{\sim}{\mathrm{K}} \mathrm{K}}_{\mathrm{K}}(\mathrm{w}-\Pi \mathrm{w}) \mathrm{ds} .
$$

Notons d'abord que par la transformation linéaire affine habituelle A de $\mathbf{K}$ en $\hat{\mathbf{K}}$, l'élément de référence, on peut définir une forme bilinéaire $\hat{\sigma}_{\mathbf{K}}$ : $\mathrm{H}^{2}(\hat{\mathbf{K}}) \times \hat{\mathbf{P}}_{a} \rightarrow \mathbb{B}$ par :

$$
\hat{\sigma}_{K}(\hat{u}, \hat{w})=h^{-1} \sigma_{K}(u, w)
$$

où

$$
\hat{\mathrm{v}}[\mathrm{A}(\underset{\sim}{\mathrm{x}})]=\mathrm{v}\left(\mathrm{x}_{\sim}^{\mathrm{x}}\right), \underset{\sim}{\mathrm{x}} \in \mathrm{K} \quad \text { et } \quad \hat{\mathrm{P}}_{a}=\left\{\hat{\mathrm{v}} / \hat{\mathrm{v}}[\mathrm{A}(\mathrm{x})]=\mathrm{v}(\underset{\sim}{\mathrm{x}}) \forall \mathrm{v} \in \mathrm{P}_{a}\right\} .
$$

vol. $17, \mathrm{n}^{\circ} 2,1983$ 
En posant

$$
\hat{\mathbf{B}}=\mathrm{A}\left(\mathrm{B}^{\mathrm{K}}\right) \quad \text { et } \quad \hat{\mathbf{F}}_{\mathrm{i}}=\mathrm{A}\left(\mathrm{F}_{\mathrm{i}}^{\mathrm{K}}\right)
$$

on peut écrire :

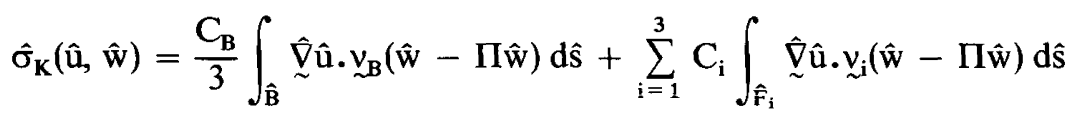

où $\left|\underline{v}_{B}\right|=\left|\underline{v}_{\mathfrak{i}}\right|=1$ et $\hat{\Pi} \hat{w}$ est le $P_{1}$-interpolé de $\hat{w}$ aux sommets de $\hat{\mathbf{K}}$.

Comme $\left\{\mathscr{C}_{\mathrm{h}}\right\}_{\mathrm{h}}$ est régulière, il existe une constante $\mathrm{C}$ indépendante de $\mathrm{h}$ telle que :

$$
\max \left(\mathrm{C}_{\mathrm{B}}, \mathrm{C}_{1}, \mathrm{C}_{2}, \mathrm{C}_{3}\right) \leqslant \mathrm{C} \quad \forall \mathrm{k} \in \mathcal{C}_{\mathrm{h}} .
$$

Ceci implique qu'il existe une constante $\hat{\mathrm{C}}$ qui ne dépend que de $\hat{\mathrm{K}}$, telle que :

$$
\left\|\hat{\sigma}_{\mathbf{K}}\right\|=\sup _{\hat{\mathbf{u}} \in \mathbf{H}^{2}(\hat{\mathrm{R}}), \hat{\mathrm{w}} \in \hat{\mathrm{P}}_{\boldsymbol{a}}} \frac{\hat{\sigma}_{\mathbf{K}}(\hat{\mathrm{u}}, \hat{\mathrm{w}})}{\|\hat{\mathbf{u}}\|_{2, \hat{\mathbf{K}}}\|\hat{\mathbf{w}}\|_{1, \hat{\mathbf{K}}}} \leqslant \hat{\mathbf{C}} \quad \forall \mathrm{W} \in \mathscr{C}_{\mathbf{h}},
$$

puisque

$$
\|\hat{\Pi} \hat{\mathbf{w}}\|_{0, \hat{\mathbf{k}}} \leqslant \hat{\mathrm{C}}^{\prime}\|\hat{\Pi} \hat{\mathrm{w}}\|_{\infty, \hat{\mathbf{K}}} \leqslant \hat{\mathrm{C}}\|\hat{\mathrm{w}}\|_{1, \hat{\mathbf{k}}}
$$

compte tenu du fait que $\hat{\mathrm{P}}_{a}$ est un espace de dimension finie.

De toute évidence on a :

$$
\hat{\sigma}_{\mathbf{K}}(\hat{\mathrm{u}}, \hat{\mathrm{w}})=0 \quad \forall \hat{\mathrm{u}} \in \mathrm{H}^{2}(\hat{\mathrm{K}}) \quad \text { et } \quad \forall \hat{\mathrm{w}} \in \mathbf{P}_{\mathrm{o}} .
$$

D'autre part, si $\hat{u} \in \mathbf{P}_{1}$, on peut dire que $\beta=\mu \underset{\sim}{\nabla u}$ est un champ de vecteurs constant sur $\mathrm{K}$, où $\mu$ est un scalaire défini par $\mathrm{w}-\Pi \mathrm{w}=\mu \varphi, \varphi$ étant donné par (3.1).

On peut donc appliquer le Lemme 3.1, ce qui entraîne :

$$
\hat{\sigma}_{\mathrm{K}}(\hat{\mathrm{u}}, \hat{\mathrm{w}})=0 \quad \forall \hat{\mathrm{u}} \in \mathrm{P}_{1} \quad \text { et } \quad \forall \hat{\mathrm{w}} \in \hat{\mathrm{P}}_{a} .
$$

On en déduit que $\hat{\sigma}_{\mathrm{K}}$ satisfait à toutes les hypothèses du Lemme 4.1, avec $\mathrm{k}=1$ et $\mathbf{l}=0$, d'où :

$$
\hat{\sigma}_{\mathbf{K}}(\hat{\mathrm{u}}, \hat{\mathrm{w}}) \leqslant \mathrm{C}|\hat{\mathrm{u}}|_{2, \mathrm{~K}}|\hat{\mathrm{w}}|_{1, \mathbf{K}} .
$$

En revenant à l'élément courant $\mathrm{K}$, par des arguments tout à fait standards on obtient :

$$
\left|\sigma_{\mathbf{K}}(\mathrm{u}, \mathrm{w})\right| \leqslant \mathrm{Ch}|\mathrm{u}|_{2, \hat{\mathbf{K}}}|\mathrm{w}|_{1, \mathbb{R}} \quad \mathrm{K} \in \mathcal{C}_{\mathbf{h}} .
$$


Compte tenu du fait que le second membre de (4.3) n'est rien d'autre que

$$
\sum_{K \in \mathcal{V}_{\mathbf{h}}} \sum_{i=1}^{3} \sigma_{K}\left(z_{i}, w_{h_{i}}\right) \text { le lemme est démontré . c.q.f.d. }
$$

On introduit maintenant $\mathrm{r}_{\mathrm{h}}: \underset{\sim}{\mathrm{W}_{\mathrm{h}}} \rightarrow{\underset{\sim}{\mathrm{h}}}_{\mathrm{W}}$, l'opérateur de projection orthogonale pour le produit scalaire $(., .)_{h}$, où $\underset{\sim}{\tilde{W}_{h}}=\underset{\sim}{V}+{\underset{\sim}{\mathrm{h}}}_{\mathrm{h}}$.

LEMME 4.3: Soit $e_{\mathrm{h}}=\underset{\sim}{\mathrm{v}}-r_{\mathrm{h}} \underset{\sim}{\mathrm{v}}$ l'erreur de la projection de $\underset{\sim}{\mathrm{v}} \in \underset{\sim}{\mathbb{W}_{\mathrm{h}}}$. On suppose que la solution du problème (4.1) appartient à $\mathrm{H}^{\mathrm{s}}(\Omega)$, avec $\mathrm{s}=2$ si $\mathrm{n}=3$ et $3 / 2-\varepsilon \leqslant \mathrm{s} \leqslant 2$ si $\mathrm{n}=2$. Alors on $a$ :

$$
|\underset{\mathrm{h}}{\mathrm{e}}|_{\mathrm{o}} \leqslant \mathrm{Ch}^{\mathrm{s}-1}|\underset{\sim}{\mathrm{v}}|_{1}
$$

Démonstration : Notons d'abord que :

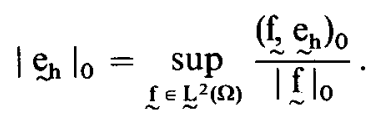

Soit $M$ l'espace $H^{\mathrm{s}}(\Omega) \cap V$. D'après les résultats d'existence pour le problème (4.1) et (4.2), en utilisant des arguments de dualité classiques, on a :

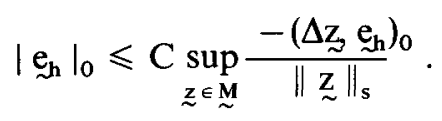

D'autre part, par application de la formule de Green au niveau de chaque $\mathrm{K} \in \mathfrak{G}_{\mathrm{h}}$ on $\mathbf{a}:$

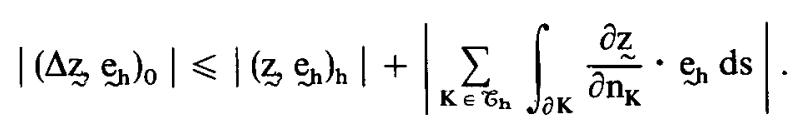

Maintenant on remarque que la somme qui figure au second membre de (4.5) s'annule pour $\mathrm{n}=2$. D'autre part, comme $\underset{\sim}{\mathrm{v}} \in \underset{\sim}{\mathrm{V}}$, pour $\mathrm{n}=3$ on a :

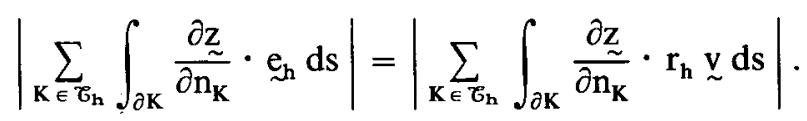

Or, d'après le Lemme 4.2 , on $\mathrm{a}$ :

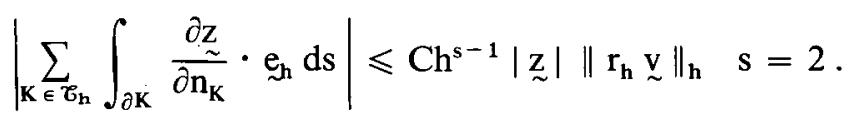

D'autre part, d'après le Théorème de l'inclusion de Sobolev [1], on doit avoir vol. $17, \mathrm{n}^{\circ} 2,1983$ 
$\underset{\sim}{z} \in \underset{\sim}{C^{0}}(\bar{\Omega})$ si s satisfait nos hypothèses. On peut donc définir $\Pi z$, et comme forcément $\prod_{\sim} \in{\underset{\sim}{h}}_{h}$ on peut écrire aussi bien pour $n=2$ que pour $n=3$ :

$$
\left|\left(z_{\sim}, \underset{\sim}{e}\right)_{h}\right|=\left|(\underset{\sim}{z}-\Pi \underset{\sim}{z} \underset{\sim}{e})_{h}\right| \leqslant|\underset{\sim}{z}-\Pi \underset{\sim}{z}|_{1}\left\|{\underset{\sim}{h}}_{h}\right\|_{h} .
$$

Or, d'après des résultats standards d'approximation (voir p. ex. [2] et [8], Vol. 2, p. 11), on a :

$$
\left|\underset{\sim}{z}-\Pi_{\sim}\right|_{1} \leqslant \mathrm{Ch}^{s-1}\|\underset{\sim}{\mathrm{z}}\|_{\mathrm{s}}
$$

ce qui, conjointement avec (4.5) et (4.6) donne :

$$
|\underset{\sim}{\mathrm{e}} \mathrm{h}| \leqslant \mathrm{Ch}^{\mathrm{s}-1}\left[\left\|{\underset{\sim}{\mathrm{h}}}_{\mathrm{h}}\right\|_{\mathrm{h}}+\|\underset{\mathrm{h}}{\mathrm{v}}\|_{\mathrm{h}}\right] .
$$

Le lemme est maintenant la conséquence triviale du fait que $\|\underset{\mathrm{h}}{\underset{\sim}{\mathrm{v}}}\|_{\mathrm{h}} \leqslant|\underset{\sim}{\mathrm{v}}|_{1}$. c.q.f.d.

LEMME 4.4 : Soit $\mathcal{C}_{\mathrm{h}}$ la partition de $\Omega$ en macrosimplexes et $\mathcal{C}_{\mathrm{h}}^{2}$ la partition compatible associée. A tout $\underset{\sim}{v} \in \underset{\sim}{\mathrm{V}}$ on peut associer $\underset{\sim}{\mathrm{V}_{\mathrm{h}}} \in{\underset{\sim}{\mathrm{h}}}_{\mathrm{h}}$ tel que :

$$
\begin{aligned}
& \sum_{\mathrm{k}=1}^{\mathrm{n}+1} \int_{\mathbf{K}_{\mathbf{k}}} \operatorname{div} \underset{\sim}{v_{\mathrm{h}}} \mathrm{d} \underset{\sim}{\mathrm{x}}=\int_{\mathbf{K}} \operatorname{div} \underset{\sim}{\mathrm{v}} \underset{\sim}{\mathrm{d}} \quad \forall \mathrm{K} \in \mathscr{C}_{\mathrm{h}} \\
& \|{\underset{\mathrm{v}}{\mathrm{h}}}_{\mathrm{h}_{\mathrm{h}}} \leqslant \mathrm{C}_{1} \mathrm{~h}^{\mathrm{s}-2}|\underset{\sim}{\mathrm{v}}|_{1} .
\end{aligned}
$$

Démonstration : On se réềre d'abord à la figure 3.3 pour la notation.

Soit $B_{k}$ la face de $K \in \mathcal{C}_{h}$ opposée au sommet $S_{k}, 1 \leqslant k \leqslant n+1$. C'est-àdire, la base de $K_{k}$. A la suite de calculs élémentaires, si $g$ est une fonction qui appartient à l'espace $\mathrm{P}_{a}$ associé $\mathrm{K}_{\mathrm{k}}$, on obtient :

$$
\int_{B_{k}} g d s=\frac{1}{6(n-1)}\left[\sum_{\substack{j=1 \\ j \neq k}}^{n+1} g\left(S_{j}\right)+(5 n-6) g\left(M_{k}\right)\right] \text { meas }\left(B_{k}\right) .
$$

On peut donc définir de manière unique un champ ${\underset{\downarrow}{h}}_{\mathfrak{h}} \in{\underset{\sim}{\mathrm{h}}}_{\mathrm{h}}$ tel que $\forall \mathrm{K} \in \mathscr{C}_{\mathrm{h}}$, on ait :

$$
\begin{gathered}
v_{h_{i}}\left(S_{j}\right)=r_{h} v_{i}\left(S_{j}\right) \quad j=1,2, \ldots, n+1 \\
\frac{5-n}{3} \int_{B_{k}} v_{h_{i}} d s+\frac{n-2}{3} \int_{B_{k}} \prod_{h_{i}} d s=\int_{B_{k}} v_{i} d s \quad k=1,2, \ldots, n+1
\end{gathered}
$$

On pose maintenant $\mu_{k}={\underset{\sim}{n} / K_{k} / B_{k}}$, on multiplie chaque membre de (4.10) par $\mu_{k_{i}}$, et on somme ensuite sur $i, 1 \leqslant i \leqslant n$. 
Pour $\mathrm{n}=2$ on obtient :

$$
\int_{B_{k}} \underset{\mathrm{h}}{\mathrm{v}_{\mathrm{h}}} \cdot \mu_{\mathrm{k}} \mathrm{ds}=\int_{\mathrm{B}_{\mathrm{k}}} \underset{\sim}{\mathrm{v}} \cdot \mu_{\mathrm{k}} \mathrm{ds}
$$

Pour $\mathbf{n}=3$ on $\mathbf{a}$ :

$$
\frac{2}{3} \int_{B_{k}}\left(v_{h}-\Pi v_{h}\right) \cdot \mu_{k} d s+\int_{B_{k}} \Pi v_{h} \cdot \mu_{j k} d s=\int_{B_{k}} \underset{\sim}{v} \cdot \mu_{k} d s .
$$

Cependant, d'après le Lemme 3.1, on $\mathrm{a}$ :

$$
\frac{2}{3} \int_{B_{k}}\left(v_{h}-\Pi v_{\mathfrak{h}}\right) \cdot \mu_{k} d s=\int_{K_{k}} \operatorname{div}\left(v_{h}-\Pi v_{h}\right) d \underset{\sim}{x} .
$$

Par conséquent, en sommant sur $k$ et en utilisant la formule de Stokes sur chaque $K_{k}$ et sur $K$ on obtient (4.7) aussi bien pour $n=2$ que pour $n=3$.

Prouvons maintenant (4.8) seulement pour $\mathrm{n}=3$. En réalité, compte tenu du Lemme 4.3, dans le cas $n=2$ cette relation peut être obtenue en tant qu'une variante triviale du Lemme 2.5 de [7].

Notons d'abord que $\left\|{\underset{\sim}{\mathrm{h}}}_{\|_{\mathrm{h}}} \leqslant\right\| \mathrm{r}_{\mathrm{h}} \underset{\sim}{\mathrm{v}}-\underline{\sim}_{\mathrm{h}} \|_{\mathrm{h}}+|\underset{\sim}{\mathrm{v}}|_{1}$. Mais

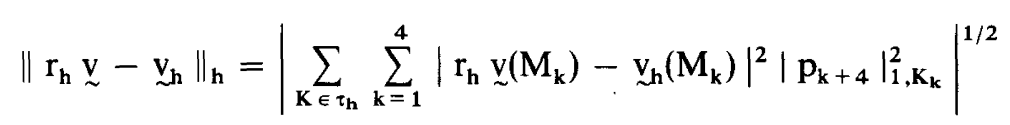

où $P_{k+4}$ est la fonction de base de $P_{a}$ associée au barycentre $M_{k}$ de $B_{k}$.

Des estimations tout à fait classiques donnent :

$$
\left|p_{k+4}\right|_{1, K_{k}} \leqslant \mathrm{Ch}^{1 / 2} \text {. }
$$

D'autre part, d'après (4.9) et (4.10) on a :

$$
\begin{gathered}
|r_{h} \underset{\sim}{v}\left(M_{k}\right)-\underbrace{}_{h}\left(M_{k}\right)|=\frac{4}{3} \frac{\left|\int_{B_{k}} r_{h} \underset{\sim}{v} d s-\int_{B_{k}}{\underset{v}{h}}_{h} d s\right|}{\int_{B_{k}} d s} \leqslant \\
\leqslant \frac{4}{3} \frac{\left|\frac{3}{2} \int_{B_{k}} e_{h} d s\right|+\frac{1}{2}\left|\int_{B_{k}}\left(r_{h} \underset{\sim}{v}-\Pi_{v_{h}}\right) d s\right|}{\int_{B_{k}} d s} .
\end{gathered}
$$


En se servant de la transformation affine $A: K_{k} \rightarrow \hat{K}$, on obtient :

$$
\left|\mathrm{r}_{\mathrm{h}} \underset{\sim}{\mathrm{v}}\left(\mathbf{M}_{\mathbf{k}}\right)-\underline{\sim}_{\mathrm{h}}\left(\mathbf{M}_{\mathbf{k}}\right)\right| \leqslant \frac{8}{3}\left[\frac{3}{2}\left|\int_{\hat{\mathrm{B}}}{\underset{\sim}{\hat{\mathrm{e}}}}_{\mathrm{h}} \mathrm{ds}\right|+\frac{1}{2}\left|\int_{\hat{\mathrm{B}}}\left(\mathrm{r}_{\mathrm{h}} \underset{\sim}{\hat{v}}-\Pi{\underset{\sim}{\mathrm{h}}}_{\mathrm{h}}\right) \mathrm{ds}\right|\right] .
$$

Mais d'après le Théorème des Traces, on a :

$$
\left|r_{h} \underset{\sim}{v}\left(M_{k}\right)-{\underset{\sim}{h}}_{h}\left(M_{k}\right)\right| \leqslant C\left[\left\|{\underset{\sim}{\hat{h}}}_{h}\right\|_{1 . \hat{K}}+\left\|r_{h} \underset{\sim}{\hat{v}}-\Pi{\underset{\sim}{h}}_{h}\right\|_{1 . \hat{K}}\right] .
$$

Comme $\hat{\Pi} \hat{\underline{v}}_{\mathbf{h}}$ interpole aussi $\mathbf{r}_{\mathrm{h}} \underset{\sim}{\hat{v}}$ aux sommets de $\hat{\mathbf{K}}$, des résultats standards d'approximation impliquent que :

$$
\left|\mathbf{r}_{\mathbf{h}} \underset{\sim}{\mathrm{v}}\left(\mathbf{M}_{\mathbf{k}}\right)-{\underset{\sim}{\mathrm{h}}}_{\mathbf{h}}\left(\mathbf{M}_{\mathbf{k}}\right)\right| \leqslant C\left[\left\|{\underset{\sim}{\mathrm{h}}}_{\mathrm{h}}\right\|_{1, \hat{\mathbf{K}}}+\left|\mathrm{r}_{\mathrm{h}} \underset{\sim}{\hat{\mathrm{V}}}\right|_{1, \mathbf{K}}\right] \text {. }
$$

En revenant à l'élément $\mathrm{K}_{\mathrm{k}}$, on obtient :

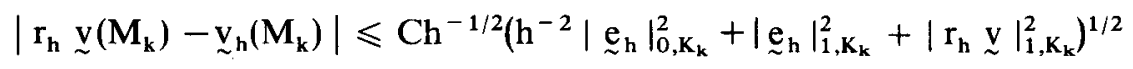

ce qui donne

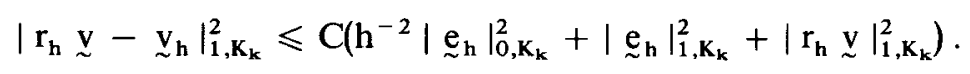

Enfin, en appliquant la sommation sur $k=1,2, \ldots, n+1$ et sur $K \in \mathscr{C}_{h}$, et en utilisant le Lemme 4.3, on obtient :

$$
\left\|\mathrm{r}_{\mathrm{h}} \underset{\sim}{\mathrm{v}}-{\underset{\sim}{\mathrm{h}}}_{\mathrm{h}}\right\|_{\mathrm{h}} \leqslant \tilde{\mathrm{C}}\left(1+\mathrm{h}^{\mathrm{s}-2}\right)\left(\left\|{\underset{\sim}{\mathrm{h}}}_{\|_{\mathrm{h}}}+\right\| \mathrm{r}_{\mathrm{h}} \underset{\sim}{\mathrm{v}} \|_{\mathrm{h}}\right),
$$

ce qui démontre (4.8) avec $\mathrm{C}_{1}=1+6 \tilde{\mathrm{C}}$. c.q.f.d.

Comme conséquence du Lemme 4.4 on a :

LEMME 4.5:Si ${\underset{\sim}{\mathrm{h}}}_{\mathrm{h}}$ est l'espace associé à $\mathfrak{C}_{\mathrm{h}}^{2}$ et $\mathrm{Q}_{\mathrm{h}}^{0}$ est l'espace de fonctions constantes sur chaque élément de $\mathcal{G}_{\mathrm{h}}$, pour un $\mathrm{q}_{\mathrm{h}} \in \mathrm{Q}_{\mathrm{h}}^{0}$ donné, il existe $\underset{\sim}{\mathrm{h}} \in \underline{\sim}_{\mathrm{h}}$ tel que :

$$
\begin{aligned}
b_{h}\left(\underline{v}_{h}, q_{h}\right) & \geqslant C_{0}\left|q_{h}\right|_{0}^{2} \\
\left\|{\underset{\sim}{h}}_{h}\right\|_{h} & \leqslant C_{2} h^{s-2}\left|q_{h}\right|_{0} .
\end{aligned}
$$

Démonstration : Soit $\mathrm{q}_{\mathrm{h}} \in \mathrm{Q}_{\mathrm{h}}^{0}$. D'après [5], Lemme $\mathrm{C}$, il existe des constantes $\mathrm{C}^{\prime}$ et $\mathrm{C}^{\prime \prime}$ indépendantes de $\mathrm{q}_{\mathrm{h}}$, ainsi que $\underset{\sim}{\mathrm{v}} \in \underset{\sim}{\mathrm{V}}$, tels que :

$$
\begin{gathered}
\mathrm{b}\left(\underset{\sim}{\mathrm{v}}, \mathrm{q}_{\mathrm{h}}\right)=|\underset{\sim}{\mathrm{v}}|_{1}^{2} \\
\mathrm{C}^{\prime}\left|\mathrm{q}_{\mathrm{h}}\right|_{0} \leqslant|\underset{\sim}{\mathrm{v}}|_{1} \leqslant \mathrm{C}^{\prime \prime}\left|\mathrm{q}_{\mathrm{h}}\right|_{0} .
\end{gathered}
$$


Comme $\mathrm{q}_{\mathrm{h}}$ est constante sur $\mathrm{K}=\bigcup_{\mathrm{k}=1}^{\mathrm{n}+1} \mathrm{~K}_{\mathrm{k}}, \mathrm{K} \in \mathcal{C}_{\mathrm{h}}$, si on associe à $\underset{\sim}{\mathrm{v}}$ le champ $\underset{\sim}{v_{h}} \in{\underset{\sim}{h}}_{\mathfrak{h}}$ satisfaisant à (4.7) et (4.8) on a :

$$
\mathrm{b}_{\mathrm{h}}\left({\underset{\sim}{\mathrm{h}}}_{\mathrm{h}}, \mathrm{q}_{\mathrm{h}}\right)=\sum_{\mathrm{K} \in \mathcal{C}_{\mathrm{k}}} \sum_{\mathrm{k}=1}^{4} \int_{\mathbf{K}_{\mathrm{k}}} \mathrm{q}_{\mathrm{h}} \operatorname{div} \underset{\sim}{\mathrm{v}} \underset{\sim}{\mathrm{d}} \mathrm{d}=\mathrm{b}\left(\underset{\sim}{\mathrm{v}}, \mathrm{q}_{\mathrm{h}}\right)
$$

ce qui implique (4.11) avec $\mathrm{C}_{0}=\left(\mathrm{C}^{\prime}\right)^{2}$.

Enfin, (4.12) est une conséquence de (4.8) avec $C_{2}=C_{1} C^{\prime \prime}$. c.q.f.d.

On introduit maintenant $\gamma$, base orthogonale de $\mathrm{Q}_{\mathrm{h}}$, avec

$$
\gamma=\bigcup_{\mathbf{K} \in \mathcal{C}_{\mathbf{h}}}\left\{\gamma_{1}^{\mathbf{K}}, \gamma_{2}^{\mathbf{K}}, \ldots, \gamma_{n+1}^{\mathbf{K}}\right\}
$$

telle que $\operatorname{supp}\left(\gamma_{\mathrm{i}}^{\mathrm{K}}\right) \subset \mathrm{K}, 1 \leqslant \mathrm{i} \leqslant \mathrm{n}+1$ et

$$
\begin{aligned}
& \text { Pour } \mathrm{n}=2 \\
& \text { Pour } \mathrm{n}=3 \\
& \gamma_{1}^{\mathbf{K}}(\underset{\sim}{\mathrm{x}})=1 \quad \forall \underset{\sim}{\mathrm{x}} \in \mathrm{K} \\
& \gamma_{1}^{\mathrm{K}}(\underset{\sim}{\mathrm{x}})=1 \quad \forall \underset{\sim}{\mathrm{x}} \in \mathbf{K} \\
& \gamma_{2}^{\mathrm{K}}(\underset{\sim}{\mathrm{x}})= \begin{cases}1 / 2 & \underset{\sim}{\forall} \in \mathrm{K}_{1} \cup \mathrm{K}_{3} \\
-1 & \forall \underset{\sim}{\forall} \in \mathrm{K}_{2}\end{cases} \\
& \gamma_{2}^{\mathbf{K}}(\underset{\sim}{\mathbf{x}})= \begin{cases}+1 & \underset{\sim}{\forall} \in \mathbf{K}_{1} \cup \mathbf{K}_{2} \\
-1 & \underset{\sim}{\forall} \in \mathbf{K}_{3} \cup \mathbf{K}_{4}\end{cases}
\end{aligned}
$$

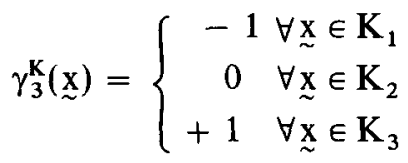

$$
\begin{aligned}
& \gamma_{3}^{\mathbf{K}}(\underset{\sim}{\mathbf{x}})= \begin{cases}+1 & \underset{\sim}{\forall} \in \mathbf{K}_{1} \cup \mathbf{K}_{3} \\
-1 & \forall \underset{\sim}{\mathbf{x}} \in \mathbf{K}_{2} \cup \mathbf{K}_{4}\end{cases} \\
& \gamma_{4}^{\mathbf{K}}(\underset{\sim}{\mathrm{x}})=\left\{\begin{array}{cc}
+1 & \forall \underset{\sim}{\forall x} \in \mathrm{K}_{1} \cup \mathbf{K}_{4} \\
-1 & \forall \underset{\sim}{x} \in \mathrm{K}_{2} \cup \mathbf{K}_{3} .
\end{array}\right.
\end{aligned}
$$

Soit $\xi_{i}^{K}$ la composante de $\mathrm{q}_{\mathrm{h}} \in \mathrm{Q}_{\mathrm{h}}$ par rapport à $\gamma_{\mathrm{i}}^{K}$. On peut donc écrire :

$$
\mathrm{q}_{\mathrm{h}}=\sum_{i=1}^{n+1} \mathrm{q}_{i}
$$

où

$$
\mathrm{q}_{\mathrm{i}}=\sum_{\mathbf{K} \in \mathcal{C}_{\mathbf{h}}} \xi_{\mathbf{i}}^{\mathbf{K}} \gamma_{\mathrm{i}}^{\mathbf{K}}, \quad 1 \leqslant \mathrm{i} \leqslant \mathrm{n}+1 .
$$

Notons que $\mathrm{Q}_{\mathrm{h}}^{0}$ est l'espace engendré par $\gamma_{1}=\bigcup_{\mathrm{K} \in \mathcal{C}_{\mathrm{h}}} \gamma_{1}^{\mathrm{K}}$.
Maintenant on prouve :

THÉORÈME 4.2 : Si on utilise la partition de type $\mathscr{C}_{\mathrm{h}}^{2}$ définie dans la Section 3, pour la construction des espaces ${\underset{\sim}{\mathrm{h}}}_{\mathrm{h}}$ et $\mathrm{Q}_{\mathrm{h}}$, alors la condition de Brezzi discrète $(2.4)_{h}$ est vérifiée avec $\beta_{h}=\beta_{0} h^{2-s}$, où $\beta_{0}$ est une constante strictement positive indépendante de $h$. 
Démonstration : Si l'espace de pressions était $\mathrm{Q}_{\mathrm{h}}^{0}$, le théorème serait démontré, puisque dans ce cas $(2.4)_{\mathrm{h}}$ ne serait qu'une conséquence immédiate de (4.11) et de (4.12). On va donc construire $\underset{\sim}{\mathrm{z}} \in \underset{\sim}{\mathrm{h}}$, un champ associé aux pressions engendrées par $\gamma_{i}^{K}, 2 \leqslant i \leqslant n+1$, afin de prouver $(2.4)_{h}$ pour l'espace $Q_{h}$ tout entier.

Pour ce faire on pose ${\underset{\sim}{i}}_{i}=\vec{S}_{i} M_{i}$, c'est-à-dire la médiane orientée de $K$ par rapport à $S_{i}, m_{i}=\left|{\underset{\sim}{i}}_{i}\right|, i=1,2, \ldots, n+1$, et

$$
q=\sum_{i=2}^{n+1} q_{i}
$$

Maintenant, sur chaque macrosimplexe $K, \underset{\sim}{z}$ sera tel que $\underset{\sim}{z} / K_{\mathbf{i}} \in \mathbf{P}_{a}$ et $\underset{\sim}{\mathbf{z}}\left(\mathrm{S}_{\mathrm{i}}\right)=\underset{\sim}{\mathbf{z}}\left(\mathbf{M}_{\mathrm{i}}\right)=\underset{\sim}{0}, 1 \leqslant \mathrm{i} \leqslant \mathrm{n}+1$.

Soit d'abord le cas $\mathbf{n}=2$ :

En supprimant l'indice $\mathrm{K}$, si $\mathrm{G}$ est le barycentre de $\mathrm{K}$, on pose :

$$
\underset{\sim}{z}(\mathrm{G})=\frac{2}{3}\left(\xi_{2} \underset{\sim}{\mathrm{m}_{2}}-\xi_{3} \underset{\sim 3}{\mathrm{~m}_{3}}\right) \text {. }
$$

On en déduit facilement que $\underset{\sim}{z} \in \underset{\sim}{\mathbf{P}}$ sur chaque $K_{\mathrm{i}}$, que $\underset{\sim}{z} \in \underset{\sim}{\mathbb{C}^{0}}(\bar{\Omega})$ et par un calcul tout à fait élémentaire on obtient (voir fig. 4.1) :

$$
|\mathrm{q}|_{0, \mathrm{~K}}^{2}=\left(\frac{3 \xi_{2}^{2}}{2}+2 \xi_{3}^{2}\right) \frac{\operatorname{mes}(\mathrm{K})}{3}
$$

ainsi que

$$
|\underset{\sim}{z}|_{1, K} \leqslant C|q|_{0, K} .
$$

Soit maintenant ${\underset{\sim}{\mathfrak{i}}}_{\mathbf{i}}$ le vecteur unitaire normal à ${\underset{\sim}{\mathfrak{i}}}_{\mathfrak{i}}$, orienté de la façon indiquée sur la figure 4.2.

On a :
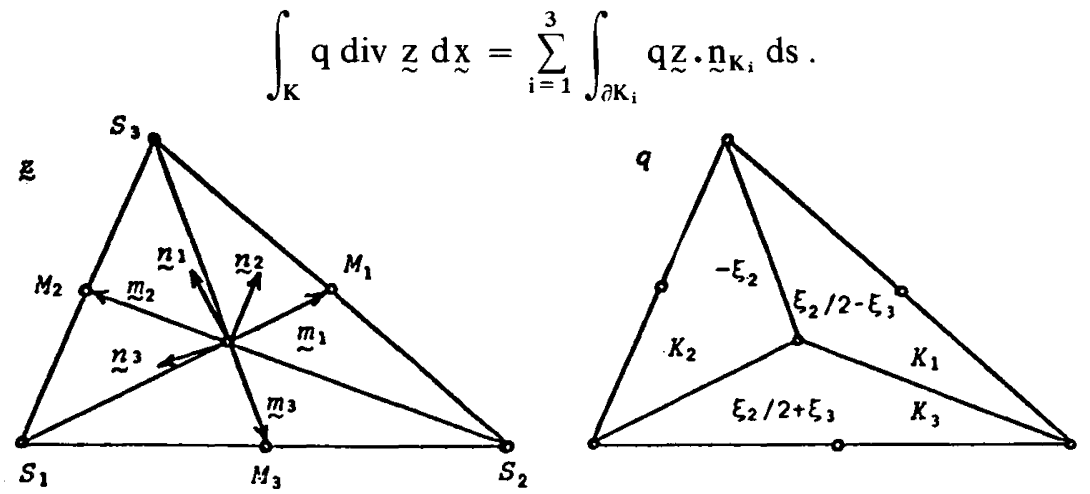

Figure 4.1. - Le champ $\underset{\sim}{z}$ associé à $q \in Q_{n}$.

R.A.I.R.O. Analyse numérique/Numerical Analysis 
ÉLÉMENTS FINIS QUASILINÉAIRES

Maintenant on utilise l'identité élémentaire :

$$
\frac{2}{3} m_{i}\left|{\underset{\sim}{j}}_{j} \cdot n_{i}\right| \equiv \operatorname{mes}(K) \quad i \neq j .
$$

Ensuite par des calculs également élémentaires on obtient :

$$
\int_{\mathrm{K}} \mathrm{q} \operatorname{div} \underset{\sim}{\mathrm{z}} \underset{\sim}{\mathrm{x}}=\frac{\operatorname{mes}(\mathrm{K})}{3}\left(3 \xi_{2}^{2}+\frac{3 \xi_{2} \xi_{3}}{2}+3 \xi_{3}^{2}\right) \geqslant|\mathrm{q}|_{0, \mathrm{~K}}^{2},
$$

ce qui donne :

$$
\mathrm{b}_{\mathrm{h}}(\underset{\sim}{\mathrm{z}}, \mathrm{q}) \geqslant|\mathrm{q}|_{0}^{2}
$$

Pour le cas $n=3$ on choisit

$$
\mathrm{z}(\mathrm{G})=\frac{3}{2}\left(-\xi_{2} \underset{\sim 2}{\mathrm{~m}_{2}}+\xi_{3} \underset{\sim 3}{\mathrm{~m}_{3}}+\xi_{4} \underset{\sim}{\mathrm{m}_{4}}\right) .
$$

Notons qu'ici aussi on a (4.13) et que

$$
|\mathrm{q}|_{0, \mathrm{~K}}^{2}=\left(\xi_{2}^{2}+\xi_{3}^{2}+\xi_{4}^{2}\right) \operatorname{mes}(\mathrm{K}) .
$$

Maintenant, grâce à l'identité :

$$
\begin{gathered}
\left|\underset{\sim \mathrm{k}}{\mathrm{m}_{\sim}} \operatorname{\sim n}_{\mathrm{ij}}\right|\left(\mathrm{F}_{\mathrm{ij}}\right) \equiv \operatorname{mes}(\mathrm{K}) \quad 1 \leqslant \mathrm{k} \leqslant 4, \quad 1 \leqslant \mathrm{i}<\mathrm{j} \leqslant 4, \quad \mathrm{i}, \mathrm{j} \neq \mathrm{k}, \\
\mathrm{i}, \mathrm{j} \neq \mathrm{k},
\end{gathered}
$$

où $\mathfrak{\sim}_{\mathrm{ij}}$ est le vecteur unitaire normal à $F_{\mathrm{ij}}$, orienté de façon convenable, $F_{\mathrm{ij}}$ étant la face dont les sommets sont $S_{j}, S_{j}$ et $G$, on déduit (4 14) à nouveau, par les mêmes arguments que dans le cas bidimensionnel.

Maintenant on remarque que

$$
\mathrm{b}_{\mathrm{h}}\left(\underset{\sim}{\mathrm{z}}, \mathrm{q}_{1}\right)=0 \quad \forall \mathrm{q}_{1} \in \mathrm{Q}_{\mathrm{h}}^{0} .
$$

D'autre part pour $\mathrm{q}_{1} \in \mathrm{Q}_{\mathrm{h}}^{0}$ donné, soit $\mathrm{y} \in{\underset{\sim}{\mathrm{h}}}_{-}$te champ qui satisfait (4.11) et (4.12).

Posons $\underset{\sim}{v_{h}}=\theta h^{2(s-2)} \underset{\sim}{z}+\underset{\sim}{y}$, où $\theta$ est un paramètre indépendant de h qui sera déterminé de telle sorte qu'il existe une constante $\mathrm{C}_{4}>0$ qui satisfait :

$$
\mathrm{b}_{\mathrm{h}}\left(\mathrm{v}_{\mathrm{h}}, \mathrm{q}_{\mathrm{h}}\right) \geqslant \mathrm{C}_{4}\left(\mathrm{~h}^{2(\mathrm{~s}-2)}|\mathrm{q}|_{0}^{2}+\left|\mathrm{q}_{1}\right|_{0}^{2}\right) .
$$

Comme

$$
b_{h}\left({\underset{\sim}{h}}_{h}, q_{h}\right)=\theta h^{2(s-2)} b_{h}(\underset{\sim}{z}, q)+b_{h}\left(\underset{\sim}{y}, q_{1}\right)+b_{h}(\underset{\sim}{y}, q)
$$

vol. $17, \mathrm{n}^{\circ} 2,1983$ 
et $b_{h}(\underset{\sim}{y}, q) \leqslant 2\|\underset{\sim}{y}\|_{h}|q|_{0}$ on a, compte tenu de (4.11), (4.12) et (4.14) :

$$
\mathrm{b}_{\mathrm{h}}\left({\underset{\sim}{\mathrm{h}}}_{\mathrm{h}}, \mathrm{q}_{\mathrm{h}}\right) \geqslant \theta \mathrm{h}^{2(\mathrm{~s}-2)}|\mathrm{q}|_{0}^{2}+\mathrm{C}_{0}\left|\mathrm{q}_{1}\right|_{0}^{2}-2 \mathrm{C}_{2} \mathrm{~h}^{\mathrm{s}-2}\left|\mathrm{q}_{1}\right|_{0}|\mathrm{q}|_{0} .
$$

Par conséquent si on choisit $\theta=4 \mathrm{C}_{2}^{2} / \mathrm{C}_{0}$, on a (4.15) avec

$$
\mathrm{C}_{4}=\min \left(\frac{2 \mathrm{C}_{2}^{2}}{\mathrm{C}_{0}}, \frac{\mathrm{C}_{0}}{2}\right) \text {. }
$$

D'autre part, d'après $(4.12)$ et $(4.13)$ on $a$ :

$$
\left|\underline{\mathrm{v}}_{\mathrm{h}}\right|_{1} \leqslant \mathrm{C}_{5} \mathrm{~h}^{\mathrm{s}-2}\left[\mathrm{~h}^{\mathrm{s}-2}|\mathrm{q}|_{0}+\left|\mathrm{q}_{1}\right|\right] .
$$

De (4.15) on déduit donc que

$$
b_{h} \frac{\left(\underline{v}_{h}, q_{h}\right)}{\left|{\underset{\sim}{h}}_{h}\right|_{1}} \geqslant C_{6} h^{2-s} \frac{h^{2(s-2)}|q|_{0}^{2}+\left|q_{1}\right|_{0}^{2}}{h^{s-2}|q|_{0}+\left|q_{1}\right|} \geqslant \frac{C_{6}}{2} h^{2-s}\left(h^{s-2}|q|_{0}+|q|_{1}\right) \text {. }
$$

En rappelant que $s \leqslant 2$, on a $\mathrm{C}_{7}=\min \left[1, \min _{\left(\boldsymbol{\vartheta}_{\mathrm{h}}\right)_{\mathrm{h}}} \mathrm{h}^{\mathrm{s}-2}\right]>0$.

Enfin, compte tenu du fait que

$$
|\mathrm{q}|_{0}+\left|\mathrm{q}_{1}\right| \geqslant\left(|\mathrm{q}|_{0}^{2}+\left|\mathrm{q}_{1}\right|_{0}^{2}\right)^{1 / 2}=\left|\mathrm{q}_{\mathrm{h}}\right|_{0}
$$

on déduit $(2.4)_{\mathrm{h}}$ avec

$$
\beta_{\mathrm{h}}=\frac{\mathrm{C}_{6} \mathrm{C}_{7}}{2} \mathrm{~h}^{2-\mathrm{s}} \quad \text { c.q.f.d. . }
$$

Le Théorème 4.2 fournit tous les outils essentiels pour l'obtention d'estimations de l'erreur dans le cas bidimensionnel. Cependant pour le cas tridimensionnel on doit établir de plus une majoration convenable pour le terme de non conformité $\mathrm{E}_{\mathrm{h}}(\underset{\sim}{\mathrm{u}} \mathrm{p}, \underset{\sim}{\mathrm{h}})$ donné par (2.6). Pour ce faire, en principe il faut traiter séparément les cas du problème $\left(\mathscr{P}_{1}\right)$ et du problème $\left(\mathscr{P}_{2}\right)$. Cependant, comme on verra dans le Lemme ci-dessous, les deux cas conduisent au même résultat, si on considère $\left(\mathscr{P}_{1}\right)$ comme une variante de $\left(\mathscr{P}_{2}\right)$ avec $\left.\Gamma_{1}=\emptyset\right)$.

LEMME 4.6 : Supposons que la solution (uv, p) de $\left(\mathscr{P}^{\prime}\right)$ appartient à

$$
\left(\underset{\sim}{\mathrm{H}^{2}}(\Omega) \cap \underset{\sim}{\mathrm{V}}\right) \times \mathrm{H}^{1}(\Omega) .
$$

Alors pour $n=3$, on $a$ :

$$
\mathrm{E}_{\mathbf{h}}(\underset{\sim}{\mathrm{u}}, \mathrm{p}, \underset{\sim \mathrm{h}}{\mathbf{w}}) \leqslant \mathrm{C}\left[\mathrm{h}\left(|\underset{\sim}{\mathbf{u}}|_{2}+|\mathbf{p}|_{1}\right)+\mathbf{h}^{1 / 2}|\underset{\sim}{\mathrm{g}}|_{0, \Gamma_{1}}\right]\left\|{\underset{\sim}{\mathrm{w}}}_{\mathrm{h}}\right\|_{\mathbf{h}} .
$$


Démonstration : On rappelle d'abord (2.11) $,(1.4)_{i}, i=1,2$ et (2.12). En appliquant la formule de Green sur chaque $K \in \mathcal{C}_{\mathrm{h}}$, on a :

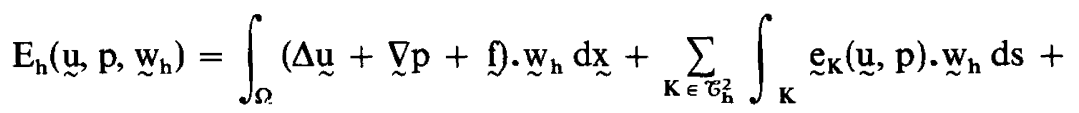

où

$$
+\int_{\Gamma_{1}} g \cdot \sim_{\sim h} d s
$$

$$
\mathrm{e}_{\mathrm{K}}(\underset{\sim}{\mathrm{u}}, \mathrm{p})=\frac{\partial \underset{\sim}{\mathrm{u}}}{\partial \mathrm{n}_{\mathrm{K}}}+\mathrm{pn}_{\sim} \quad \text { pour } \quad\left(\mathscr{P}_{1}\right)
$$

et

$$
\mathrm{e}_{\mathrm{K}}(\underset{\sim}{\mathrm{u}}, \mathrm{p})=\underset{\sim}{\varepsilon}(\underset{\sim}{\mathrm{u}}) \cdot{\underset{\sim}{\mathrm{K}}}_{\mathrm{K}}+\mathrm{pp}_{\sim} \mathrm{K} \text { pour }\left(\mathscr{P}_{2}\right) .
$$

En rappelant que pour les deux cas $\underset{\sim}{\mathrm{u}}+\underset{\sim}{\nabla} \mathrm{p}+\underset{\sim}{\mathrm{f}}=\underset{\sim}{0}$, que $\underset{\sim}{\mathrm{e}} \mathbf{K}(\underset{\sim}{\mathrm{u}}, \mathrm{p})=\mathbf{g}$ sur toute face contenue dans $\Gamma_{1}$, et que $\tilde{\sim}_{\mathrm{h}}$ est continue sur les bases de $\mathcal{G}_{\mathrm{h}}^{2}$, on a :

$$
\mathrm{E}_{\mathrm{h}}\left(\underset{\sim}{\mathrm{u}}, \mathrm{p}, \underset{\sim}{w_{h}}\right)=\sum_{\mathbf{K} \in \mathscr{C}_{\mathrm{h}}^{2}} \sum_{\mathrm{i}=1}^{3} \int_{F_{i}^{K}}{\underset{\mathrm{e}}{\mathrm{K}}}_{\mathrm{K}}(\underset{\sim}{\mathrm{u}}, \mathrm{p}) \cdot \underset{\sim}{w_{h}} \mathrm{ds} .
$$

Prenons maintenant le cas de $\left(\mathscr{P}_{2}\right)$.

Comme ${\underset{\sim}{\mathrm{h}}}_{\mathrm{h}}$ est continue sur les bases $\mathrm{B}^{\mathrm{K}}$, ainsi que $\Pi_{\sim_{h}}$ sur toute face de $\mathcal{G}_{h}^{2}$, en procédant comme dans le Lemme 4.2 , on peut réécrire $E_{h}(\underset{\sim}{u}, p, \underset{\sim}{w})$ de la manière suivante :

$$
\begin{aligned}
& \mathrm{E}_{\mathrm{h}}\left(\underset{\sim}{\mathrm{u}}, \mathrm{p},{\underset{\sim}{\mathrm{w}}}_{\mathrm{h}}\right)=\sum_{\mathrm{K} \in \mathcal{C}_{\mathrm{h}}^{2}}\left\{\sum_{\mathrm{i}=1}^{3} \tau_{\mathrm{K}}\left[{\underset{\sim}{\mathrm{i}}}_{\mathrm{i}}(\underset{\sim}{\mathrm{u}}), \mathrm{w}_{\mathbf{h}_{\mathrm{i}}}\right]+\zeta_{\mathrm{K}}\left(\mathrm{p}, \underset{\sim \mathrm{h}}{\mathrm{w}_{\mathrm{h}}}\right)\right\}- \\
& -\frac{1}{3} \int g \cdot\left(\sim_{h}-\Pi{\underset{\sim}{h}}_{h}\right) d s
\end{aligned}
$$

où

$$
\underbrace{}_{\mathrm{i}}(\mathrm{u})=\left[\varepsilon_{\mathrm{i} 1}(\underset{\sim}{\mathrm{u}}), \varepsilon_{\mathrm{i} 2}(\underset{\sim}{\mathrm{u}}), \varepsilon_{\mathrm{i} 3}(\underset{\sim}{\mathrm{u}})\right] \quad \text { et } \quad \tau_{\mathrm{K}}: \underset{\sim}{\mathrm{H}^{1}}(\mathrm{~K}) \times \mathrm{P}_{a} \rightarrow \mathbb{R}
$$

et

$$
\zeta_{\mathbf{K}}: \mathrm{H}^{1}(\mathrm{~K}) \times \underset{\sim}{\mathbf{P}} \rightarrow \mathbb{R}
$$

sont donnés respectivement par :

$$
\begin{aligned}
& \tau_{\mathrm{K}}(\underset{\sim}{\mathrm{z}}, \mathrm{w})=\sum_{\mathrm{i}=1}^{3} \int_{\mathrm{F}_{\mathrm{i}}^{\mathrm{K}}} \underset{\sim}{\mathrm{z}} \cdot{\underset{\sim}{\mathrm{K}}}_{\mathrm{K}}(\mathrm{w}-\Pi \mathrm{w}) \mathrm{ds}+\frac{1}{3} \int_{\mathrm{B}^{\mathrm{K}}} \underset{\sim}{\mathrm{z}} \cdot \underset{\sim}{n_{\mathrm{K}}}(\mathrm{w}-\Pi \mathrm{w}) \mathrm{ds} \\
& \zeta_{\mathrm{K}}(\mathrm{p}, \underset{\sim}{\mathrm{w}})=\sum_{\mathrm{i}=1}^{3} \int_{\mathrm{F}_{\mathrm{i}}^{\mathrm{K}}} \mathrm{p}_{\sim} \mathrm{K}_{\mathrm{K}} \cdot\left(\underset{\sim}{\mathrm{w}}-\Pi_{\sim}^{\mathrm{w}}\right) \mathrm{ds}+\frac{1}{3} \int_{\mathrm{B}^{\mathrm{K}}} \mathrm{p}_{\sim} \mathrm{\sim}_{\mathrm{K}} \cdot\left(\underset{\sim}{\mathrm{w}}-\Pi_{\sim}^{\mathrm{w}}\right) \mathrm{ds} .
\end{aligned}
$$


Or, on constate que $\tau_{\mathrm{K}}$ et $\zeta_{\mathrm{K}}$ peuvent être traités, pour l'essentiel, comme $\sigma_{\mathrm{K}}$ dans le Lemme 4.2, ce qui permet de conclure que

$$
\tau_{\mathrm{K}}(\underset{\sim}{\mathrm{z}}, \mathrm{w}) \leqslant \mathrm{Ch}|\underset{\sim}{\mathrm{z}}|_{1, \mathrm{~K}}|\mathrm{w}|_{1, \mathrm{~K}} \quad \forall(\underset{\sim}{\mathrm{z}}, \mathrm{w}) \in \underset{\sim}{\mathrm{H}^{1}}(\mathrm{~K}) \times \mathrm{P}_{a}
$$

et

$$
\zeta_{\mathbf{K}}(\mathrm{p}, \underset{\sim}{\mathrm{w}}) \leqslant \mathrm{Ch}|\mathrm{p}|_{1, \mathrm{~K}}|\underset{\sim}{\mathrm{w}}|_{1, \mathbf{K}} \quad \forall(\mathrm{p}, \underset{\sim}{\mathrm{w}}) \in \mathrm{H}^{1}(\mathrm{~K}) \times \underset{\sim}{\mathrm{P}} .
$$

Comme

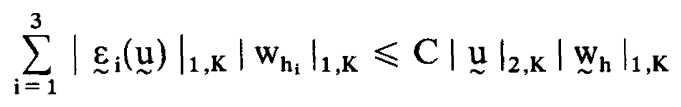

on obtient pour le cas de $\left(\mathscr{P}_{2}\right)$ :

$$
\mathrm{E}_{\mathrm{h}}\left(\underset{\sim}{\mathrm{u}}, \mathrm{p},{\underset{\sim}{\mathrm{w}}}_{\mathrm{h}}\right) \leqslant \mathrm{Ch}\left[|\underset{\sim}{\mathrm{u}}|_{2}+|\mathrm{p}|_{1}\right]+\left|\frac{1}{3} \int_{\Gamma_{1}} \underset{\sim}{\mathrm{g}} \cdot\left({\underset{\sim}{\mathrm{h}}}_{\mathrm{h}}-\Pi \underset{\sim}{\mathrm{h}}\right) \mathrm{ds}\right| .
$$

Notons que pour le cas de $\left(\mathscr{P}_{1}\right)$ on obtiendrait à nouveau (4.17), en faisant des modifications mineures dans l'analyse ci-dessus (avec $\Gamma_{1}=\emptyset$ ).

Maintenant tout ce qui reste à faire c'est donc d'estimer l'intégrale sur $\Gamma_{1}$ de (4.17). On a :

$$
\int_{\Gamma_{1}} g \cdot\left(w_{h}-\Pi_{\sim} w_{h}\right) d s=\sum_{B \subset \Gamma_{1}} \int_{B} g \cdot\left(w_{h}-\Pi_{\sim} w_{h}\right) d s
$$

où $\mathrm{B}$ dénote une base de $\mathscr{C}_{\mathrm{h}}^{2}$.

Soit $\eta_{\mathrm{K}}: \mathrm{P}_{a} \rightarrow \mathbb{R}$ défini par :

$$
\eta_{K}(w)=\int_{B} g(w-\Pi w) d s, \text { où } g \in L^{2}(B) .
$$

En appliquant la transformation affine habituelle $A: K \rightarrow \hat{K}$, on définit $\hat{\eta}: \hat{\mathbf{P}}_{a} \rightarrow \mathbf{R}$ par :

$$
\hat{\eta}=\int_{\mathbf{B}} \hat{\mathrm{g}}(\hat{\mathrm{w}}-\Pi \hat{\mathrm{w}}) \mathrm{d} \hat{\mathbf{s}}
$$

De toute évidence on a :

$$
\left|\eta_{\mathrm{K}}(\mathrm{w})\right| \leqslant \mathrm{Ch}^{2}|\hat{\eta}(\hat{\mathrm{w}})| \mathrm{K} \in \mathcal{C}_{\mathrm{h}}^{2},
$$

et

$$
\|\hat{\eta}\|=\sup _{\hat{\mathbf{w}} \in \hat{\mathbf{P}}_{a}} \frac{\hat{\eta}(\hat{\mathbf{w}})}{\|\hat{\mathbf{W}}\|_{1, \mathbb{R}}} \leqslant C|\hat{\mathbf{g}}|_{0, \hat{\mathbf{B}}}
$$


On remarque maintenant que $\hat{\eta}(\hat{w})=0 \quad \forall \hat{w} \in P_{1}, 1=0$ ou 1 . Donc en appliquant le Lemme de Bramble-Hilbert, on obtient :

$$
|\hat{\eta}(\hat{\mathrm{w}})| \leqslant \hat{\mathrm{C}}|\hat{\mathrm{g}}|_{0, \hat{\mathbf{B}}}|\hat{\mathrm{w}}|_{1, \mathbf{K}}
$$

ce qui par des transformations tout à fait standards donne :

$$
\left|\eta_{\mathbf{K}}(\mathrm{w})\right| \leqslant \mathrm{Ch}^{1 / 2}|\mathrm{~g}|_{0, \mathbf{B}}|\mathrm{w}|_{1, \mathbf{K}} .
$$

Enfin en posant successivement $g=g_{i}$ et $w=w_{h_{i}}, i=1,2,3$, on obtient :

$$
\int_{\Gamma_{1}} \underset{\sim}{g} \cdot\left(\underset{\sim}{w_{h}}-\Pi \underset{\sim}{w}\right) d s \leqslant C^{1 / 2}|\underset{\sim}{g}|_{0, \Gamma_{1}}\left\|{\underset{\sim}{h}}_{h}\right\|_{h},
$$

ce qui, compte tenu de (4.17), démontre le Lemme. c.q.f.d.

Remarque : Avec les mêmes arguments de la démonstration du Lemme 4.6 et les résultats de [19] pour l'approximation de problèmes aux limites elliptiques, on prouverait que, si le problème

$$
\left\{\begin{array}{l}
\text { Trouver } z \in \mathrm{V} \text { tel que } \\
(\underset{\sim}{\nabla \mathrm{z}}, \underset{\sim}{\nabla \mathrm{v}})_{0}=(\mathrm{f}, \mathrm{v})_{0}+(\mathrm{g}, \mathrm{v})_{0, \Gamma_{1}} \quad \forall \mathrm{v} \in \mathrm{V}
\end{array}\right.
$$

est approché par

$$
\left\{\begin{array}{l}
\text { Trouver } \mathrm{z}_{\mathrm{h}} \in \mathrm{V}_{\mathrm{h}} \text { tel que } \\
\left(\mathrm{z}_{\mathrm{h}}, \mathrm{v}_{\mathrm{h}}\right)_{\mathrm{h}}=\left(\mathrm{f} . \mathrm{v}_{\mathrm{h}}\right)_{0}+\left(\mathrm{g}, \mathrm{v}_{\mathrm{h}}\right)_{0, \Gamma_{1}} \quad \forall \mathrm{v}_{\mathrm{h}} \in \mathrm{V}_{\mathrm{h}}
\end{array}\right.
$$

on aurait l'estimation d'erreur suivante :

$$
\left\|\mathrm{z}-\mathrm{z}_{\mathrm{h}}\right\|_{\mathrm{h}} \leqslant \mathrm{Ch}|\mathrm{z}|_{2}+\overline{\mathrm{Ch}}^{1 / 2}|\mathrm{~g}|_{0, \Gamma_{1}},
$$

dont l'optimalité a été confirmée par des tests numériques.

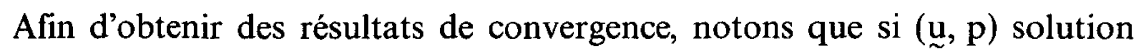
de $\left(\mathscr{P}^{\prime}\right)$ est telle que $\underset{\sim}{\mathrm{u}} \in{\underset{\sim}{\mathrm{H}}}^{\mathrm{d}}(\Omega)$, on a $\mathrm{p} \in \mathrm{H}^{\mathrm{d}-1}(\Omega)$. D'autre part, comme $\mathrm{P}_{1} \subset \mathrm{P}_{a} \subset \mathrm{P}_{2}$, si on suppose que $1 \leqslant \mathrm{~d} \leqslant 2$, on peut appliquer des résultats classiques d'approximation (voir par exemple [2] et [8], Vol. 2, p. 16), d'où on déduit :

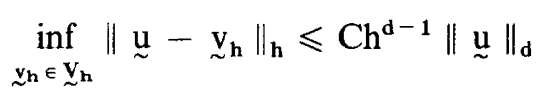

$$
\begin{aligned}
& \inf _{q_{h} \in Q_{h}}\left|p-q_{h}\right|_{0} \leqslant C^{d-1}\|p\|_{d-1} \text {. }
\end{aligned}
$$

vol. $17, \mathrm{n}^{\circ} 2,1983$ 
Enfin, en rappelant (2.7) et (2.8), on obtient les estimations de l'erreur suivantes, qui s'appliquent au cas où $\left(\mathscr{P}^{\prime}\right)$ est approché par $\left\{\left(\mathscr{P}_{\mathrm{h}}^{\prime}\right)\right\}_{\mathrm{h}}$, où $\left\{V_{h}\right\}_{h}$ et $\left\{Q_{h}\right\}_{h}$ sont des familles d'espaces associées à une famille de partitions régulières $\left\{\mathcal{G}_{\mathrm{h}}^{2}\right\}_{\mathrm{h}}$, définie à la Section 3 .

THÉORÈME 4.3 : Soit $\mathrm{n}=2$ et $\Gamma_{0}$ et $\Gamma_{1}$ tels que la solution du problème (4.1) appartient à $\mathrm{H}^{\mathrm{s}}(\Omega), 3 / 2-\varepsilon \leqslant \mathrm{s} \leqslant 2$, et la solution ( $\left.\underset{\sim}{\mathrm{u}}, \mathrm{p}\right)$ de $\left(\mathscr{P}^{\prime}\right)$ appartient à $\underset{\sim}{H^{\mathrm{d}}}(\Omega) \times H^{\mathrm{d}-1}(\Omega), 1 \leqslant \mathrm{~d} \leqslant 2$. Alors la solution $\left(\underset{\mathrm{u}}{\mathrm{h}}, \tilde{\mathrm{p}}_{\mathrm{h}}\right)$ de $\left(\mathscr{P}_{\mathrm{h}}^{\prime}\right)$ satisfait :

$$
\begin{aligned}
\left|\underset{\sim}{u}-{\underset{\sim}{\mathrm{h}}}_{\mathrm{h}}\right|_{1} \leqslant \mathrm{C}\left[\mathrm{h}^{\mathrm{d}+\mathrm{s}-3}\|\underset{\sim}{\underset{u}{u}}\|_{\mathrm{d}}+\mathrm{h}^{\mathrm{d}-1}\|\mathrm{p}\|_{\mathrm{d}-1}\right] \\
\left|\mathrm{p}-\mathrm{p}_{\mathrm{h}}\right|_{0} \leqslant \mathrm{C}\left[\mathrm{h}^{\mathrm{d}+2 \mathrm{~s}-5}\|\underset{\sim}{\mathrm{u}}\|_{\mathrm{d}}+\mathrm{h}^{\mathrm{d}+\mathrm{s}-3}\|\mathrm{p}\|_{\mathrm{d}-1}\right] .
\end{aligned}
$$

THÉORÈME 4.4 : Soit $\mathbf{n}=3$ et $\Gamma_{0}$ et $\Gamma_{1}$ tels que la solution du problème (4.1) appartient à $\mathrm{H}^{2}(\Omega)$ et la solution de $\left(\mathscr{P}^{\prime}\right)$ appartient à $\underset{\sim}{\mathrm{H}^{2}}(\Omega) \times \mathrm{H}^{1}(\Omega)$. Alors la solution $\left(\mathrm{u}_{\mathrm{h}}, \mathrm{p}_{\mathrm{h}}\right)$ de $\left(\mathscr{P}_{\mathrm{h}}^{\prime}\right)$ satisfait :

$\left\|\underset{\sim}{\mathrm{u}}-{\underset{\sim}{\mathrm{h}}}_{\mathrm{u}}\right\|_{\mathrm{h}}+\left|\mathrm{p}-\mathrm{p}_{\mathrm{h}}\right|_{0} \leqslant \mathrm{Ch}\left[|\mathbf{u}|_{2}+|\mathrm{p}|_{1}\right]+\overline{\mathbf{C}} \mathrm{h}^{1 / 2}|\mathrm{~g}|_{0, \Gamma_{1}}$.

Il reste à interpréter $(4.18) \sim(4.20)$ pour les cas de $\left(\mathscr{P}_{1}\right)$ et de $\left(\mathscr{P}_{2}\right)$ séparément.

Tout d'abord on remarque que pour $\left(\mathscr{P}_{1}\right)$ on peut prendre $\mathrm{s}=\mathrm{d}=2$ si $\Omega$ est convexe [13]. Compte tenu du fait que dans ce cas $\Gamma_{1}=\emptyset$, ceci implique que, aussi bien pour $\mathrm{n}=2$ que pour $\mathrm{n}=3$, la majoration d'erreur optimale $\operatorname{Ch}\left[|\underset{\sim}{\mathfrak{u}}|_{2}+|\mathrm{p}|_{1}\right]$ est atteinte. Notons que, si on compare ce résultat avec celui du Théorème 4.1 pour $n=2$, on déduit que la partition $\mathscr{C}_{h}^{2}$ est supérieure à $\mathfrak{C}_{\mathrm{h}}^{1}$ sur le plan de la convergence.

Pour ce qui est du problème $\left(\mathscr{P}_{2}\right)$ on remarque d'abord que l'erreur en $\mathrm{O}(\mathrm{h})$ pour $\mathrm{n}=3$ ne peut être atteinte que s'il n'y a pas de charges surfaciques. De plus, tant en dimension deux qu'en dimension trois, la régularité $\mathrm{H}^{2}(\Omega) \times \mathrm{H}^{1}(\Omega)$ de (u,, $\left.\mathrm{p}\right)$ et la régularité $\mathrm{H}^{2}(\Omega)$ pour la solution $\mathrm{z}$ de $(4.1)$ ne s'applique que lorsque $\bar{\Gamma}_{0}$ et $\bar{\Gamma}_{1}$ sont disjointes, ce qui revient à dire que $\Gamma_{0}$ est située à l'intérieur de $\Gamma_{1}$ et vice versa.

Regardons maintenant en détail le cas bidimensionnel, puisque le Théorème $4.3 \mathrm{a}$ un champ d'application plus ample que le Théorème 4.4. Pour ce faire supposons que $s=d$, ce qui est une hypothèse raisonnable.

Tout d'abord on remarque que si $s<3 / 2$ on ne peut pas s'assurer que les déplacements convergent dans $\underset{\sim}{V}$. Ceci exclut immédiatement le cas où $\bar{\Gamma}_{0}$ et $\bar{\Gamma}_{1}$ s'intersectent en un point qui n'est pas un sommet de $\Omega$, compte tenu des résultats de régularité de la solution de (4.1) [9]. Autrement les déplacements approchés convergent vers $\underset{\sim}{\mathrm{u}}$ dans $\underset{\sim}{\mathrm{V}}$, dès que les angles des coins de $\Omega$ où 
$\bar{\Gamma}_{1}$ intersecte $\Gamma_{0}$ soient inférieurs à $\pi$. En particulier, si aucun de ces angles n'est supérieur à $\pi / 2$, on $\mathrm{a}$ :

$$
\left|\underset{\sim}{\mathbf{u}}-{\underset{\sim}{\mathrm{h}}}_{\mathrm{h}}\right|_{1} \leqslant \mathrm{Ch}^{1-\varepsilon}\left[\|\underset{\sim}{\mathbf{u}}\|_{2-\varepsilon}+\|\mathrm{p}\|_{1-\varepsilon}\right] .
$$

Par exemple, si $\Omega$ est un rectangle qui a un côté fixé ou plus, on a des résultats de convergence pratiquement optimaux.

Pour ce qui est de la pression, la condition de convergence est plus stricte, puisqu'il faut qu'on ait $\mathrm{s}<5 / 3$. Ceci correspond à une borne supérieure de $3 \pi / 4$ pour les angles des coins de $\Omega$ où $\bar{\Gamma}_{0}$ intersecte $\bar{\Gamma}_{1}$.

\section{REMARQUES COMPLÉMENTAIRES}

(i) Comme on a signalé dans la section précédente, la partition $\mathcal{C}_{\mathrm{h}}^{2}$ est plus intéressante que la partition $\mathcal{C}_{\mathrm{h}}^{1}$, si on veut s'assurer des meilleures propriétés de convergence qu'on peut obtenir avec nos éléments. Cependant il est clair que, pour un degré de raffinement de maillage donné, la première partition entraînera un volume de calcul plus important, puisqu'il y aura plus de points de calcul que pour $\mathcal{C}_{\mathbf{h}}^{1}$. C'est pour cette raison qu'il est recommandé d'effectuer une réduction de la taille de la matrice associé au problème $\left(\mathscr{P}_{h}^{\prime}\right)$, lorsqu'on travaille avec la partition $\mathscr{C}_{\mathrm{h}}^{2}$. Ceci peut être accompli en remarquant que les composantes de déplacements aux nœuds barycentriques d'un macrosimplexe de la partition associée $\mathscr{C}_{\mathrm{h}}$, sont des degrés de liberté qui peuvent être exprimés en fonction des seuls $2 \mathrm{n}(\mathrm{n}+1)$ degrés de liberté voisins, à savoir, ceux attachés à la frontière du macrosimplexe. Ceci permet donc d'éliminer les lignes de la matrice qui correspondent à ces degrés de liberté barycentriques, quitte à substituer dans les autres lignes ses valeurs en fonction des degrés de liberté voisins et à y changer le second membre en conséquence.

Notons que, sur le plan du calcul, pour le cas bidimensionnel, ce procédé équivaut à travailler avec un élément pour lequel le déplacement (vitesse) est de type $\bar{P}_{2}$ continu et la pression est de type $P_{1}$ discontinue, sur chaque triangle de $\mathfrak{C}_{\mathrm{h}}$. Ce faisant, on aurait les mêmes résultats de convergence que pour l'élément de Fortin [6], où la vitesse est aussi de type $P_{2}$ continue, alors que la pression est de type $P_{0}$ discontinue sur chaque triangle de $\boldsymbol{C}_{\mathrm{h}}$. Cependant dans le cas de notre élément la pression - et donc la condition d'incompressibilité - est approchée de manière plus fine.

Dans le cas tridimensionnel, la remarque ci-dessus s'applique au cas d'un élément proposé par l'auteur dans [17], qui généralise l'élément de Fortin. Les espaces $V_{h}$ et $Q_{h}$ qui lui sont associés, sont définis de la manière suivante (on se réfère à la figure 5.1) : 
- $\mathrm{V}_{\mathrm{h}}$ est l'espace de fonctions continues aux sommets et aux barycentres des faces des tétraèdres $K \in \mathscr{C}_{\mathbf{h}}$, dont la restriction à chaque $\mathrm{K}$ appartient à l'espace $P_{5 / 3}, P_{1} \subset P_{5 / 3} \subset P_{2}$, engendré par $\lambda_{i}, \lambda_{i}^{2}, i=1,2,3,4$.

- $Q_{h}$ est l'espace de fonctions constantes sur chaque $K \in \mathcal{C}_{\mathrm{h}}$.

L'élément ainsi défini, qui est lui aussi non conforme en déplacements, a été étudié dans [17]. Les résultats de convergence qu'on y obtient sont tout à fait équivalents à ceux qui s'appliquent au cas de l'élément de Fortin, et à l'élément $\mathrm{P}_{a} \times \mathrm{P}_{0}$ pour la partition $\mathcal{C}_{\mathrm{h}}^{2}$, dans le cas d'un problème de Stokes, à savoir :

$$
\left\|\underset{\sim}{\mathrm{u}}-{\underset{\sim}{\mathrm{h}}}_{\mathrm{h}}\right\|_{\mathrm{h}}+\left|\mathrm{p}-\mathrm{p}_{\mathrm{h}}\right|_{0} \leqslant \mathrm{Ch}\left[|\underset{\sim}{\mathrm{u}}|_{2}+|\mathrm{p}|_{1}\right] .
$$

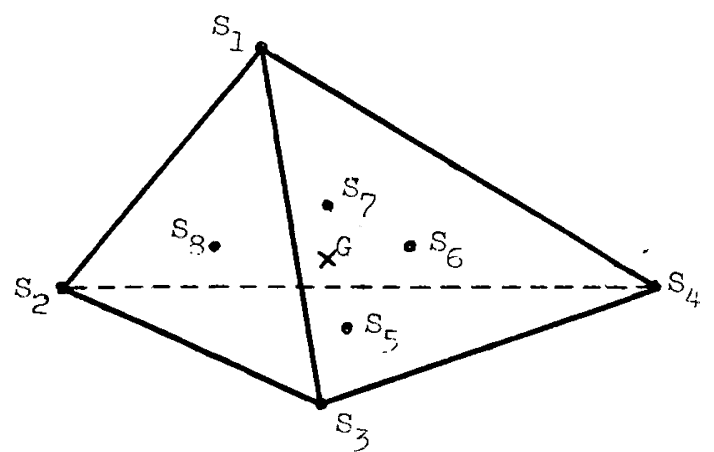

Figure 5.1. - L'élément $P_{5 / 3} \times P_{0}$.

Notons que, tout comme dans le cas bidimensionnel, le procédé qui consiste à éliminer les degrés de liberté déplacements barycentriques au niveau de la matrice, équivaut à calculer avec l'espace $V_{h}$ défini ci-dessus et l'espace $Q_{h}$ constitué de pressions de type $\mathrm{P}_{1}$ discontinues.

(ii) Les éléments asymétriques étudiés dans cet article ont d'autres propriétés intéressantes. En particulier ils permettent d'approcher de façon très efficace les problèmes d'élasticité où la condition d'incompressibilité non linéaire intervient, c'est-à-dire, le cas où $\mathrm{H}(\underset{\sim}{\mathrm{v}})=\mathrm{J}[\underset{\sim}{\mathrm{x}}+\underset{\sim}{\mathrm{v}}(\underset{\sim}{\mathrm{x}})]-1=0$ p.p. dans $\Omega$.

En effet, on prouve dans [18] que si $\underset{\sim}{\mathrm{v}} \in \underset{\sim}{\mathrm{P}_{a}}$, le Jacobien ci-dessus est un polynôme de degré un et non pas un polynôme de degré n, comme c'est le cas de champs $\underset{\sim}{\mathrm{v}}$ quadratiques en général. Cette propriété permet de simuler de façon très stable et précise le phénomène de l'incompressibilité, notamment parce que dans ce cas $\mathrm{H}(\underset{\sim}{\mathrm{v}})$ a la même forme que dans le cas $\mathrm{H}(\underset{\sim}{\mathrm{v}})=\operatorname{div} \underset{\sim}{\mathrm{v}}$ étudié dans cet article. 
Plus de détails sur ce cas non linéaire, y compris des exemples numériques, peuvent être trouvés dans [18], alors que des résultats plus récents feront l'objet d'un article de prochaine parution.

\section{REMERCIEMENTS}

L'auteur tient à remercier vivement M. R. Glowinski de l'avoir accueilli chaleureusement à l'INRIA, où ce travail a été mis au point, et à M. J. M. Thomas pour de fructueuses discussions.

\section{REFERENCES}

[1] R. A. Adams, Sobolev Spaces, Academic Press, New York, 1975.

[2] A. K. AzIZ and I. BabUSKA, Survey lectures on the mathematical foundations of the finite element method, in : The Mathematical Foundations of the Finite Element Method with Applications to Partial Differential Equations, edited by by A. K. Aziz, Academic Press, New York, 1972, pp. 3-359.

[3] F. BrezzI, On the existence, uniqueness and approximation of saddle-point problems arising from Lagrange multipliers, RAIRO Analyse Numérique 8-R2, 1974, pp. 129-151.

[4] P. G. Ciarlet, The Finite Element Method for Elliptic Problems, North Holland, Amsterdam, 1978.

[5] J. F. DebongNie, Sur la formulation de Herrmann pour l'étude des solides incompressibles, Journal de Mécanique, Vol. 17, $\mathrm{n}^{\circ} 4$, 1978, pp. 531-557.

[6] M. Fortin, Calcul Numérique des Écoulements de Fluides de Bingham et des Fluides Incompressibles par la méthode des Éléments Finis, Thèse de Doctorat d'État ès Sciences, Université Paris VI, 1972.

[7] V. Girault and P. A. Raviart, Finite Element Approximation of the NavierStokes Equations, Lecture notes in Mathematics, Springer Verlag, Berlin, 1979.

[8] R. Glowinski, J. L. LIONs and R. Trémolières, Analyse Numérique des Inéquations Variationnelles, Éditions Dunod-Bordas, Paris, 1976.

[9] P. Grisvard, Behavior of the solutions of an elliptic boundary value problem in a polygonal or a polyhedral domain, in : Numerical Solution of Partial Differential Equations, III SYNSPADE 1975, edited by B. Hubbard, Academic Press, New York, 1976.

[10] C. Johnson and J. Pitkäranta, Analysis of some mixed finite element methods related to reduced integration, Research Report $80.02 \mathrm{R}$ of the Department of Computer Sciences of the Chalmers University of Technology and the University of Göteborg, 1980.

[11] O. A. Ladyzhenskaya and N. N. Ural'Ceva, Équations aux Dérivées Partielles de type Elliptique, Dunod, Paris, 1968.

[12] T. ODEn, Finite Elements of Nonlinear Continua, McGraw Hill, New York, 1972.

[13] J. E. OsBoRH, Regularity of Solutions of the Stokes problem in a polygonal domain, in : Numerical Solution of Partial Differential Equations, III Synspade 1975, edited by B. Hubbard, Academic Press, New York, 1976.

[14] J. PItKaränta, On a mixed finite element method for the Stokes problem in $\mathbb{R}^{3}$, RAIRO - Analyse Numérique (à paraître).

vol. $17, \mathrm{n}^{\circ} 2,1983$ 
[15] G. Raugel, Résolution Numérique de Problèmes Elliptiques dans des domaines avec Coins, Thèse de Doctorat de Troisième Cycle, Université de Rennes, 1978.

[16] V. RUAS, A class of asymmetric finite element methods for solving finite incompressible elasticity problems, Comp. Meths. in Appl. Mechs. and Engin., 27, 1981, pp. 319-343.

[17] V. RUAS, Une méthode d'éléments finis non conformes en vitesse pour le problème de Stokes tridimensionnel, Matematica Aplicada e Computacional, V. 1, 1, pp. 53-74, 1982.

[18] V. Ruas, Méthodes d'Éléments Finis en Élasticité Incompressible Non Linéaire et Diverses Contributions à l'Approximation des Problèmes aux Limites, Thèse de Doctorat d'État ès Sciences, Université Pierre et Marie Curie, Paris, janvier 1982.

[19] G. Strang and J. FIx, An Analysis of the Finite Element Method, Prentice Hall, Englewood Cliffs, N.J., 1973.

[20] R. Temam, Navier-Stokes Equations, North Holland, Amsterdam, 1977.

[21] J. M. Thomas, Sur l'Analyse Numérique de Méthodes d'Éléments Finis Hybrides et Mixtes, Thèse de Doctorat d'État ès Sciences, Université Pierre et Marie Curie, Paris, 1977. 